Epidemiology and Psychiatric

Sciences

cambridge.org/eps

\section{Original Article}

Cite this article: Xue WQ, Cheng KK, Xu D, Jin X, Gong WJ (2020). Uptake of referrals for women with positive perinatal depression screening results and the effectiveness of interventions to increase uptake: a systematic review and meta-analysis. Epidemiology and Psychiatric Sciences 29, e143, 1-17. https:// doi.org/10.1017/S2045796020000554

Received: 14 February 2020

Revised: 5 June 2020

Accepted: 21 June 2020

Key words:

Depression; mental health; systematic reviews; women

Author for correspondence:

Wenjie Gong,

E-mail: gongwenjie@csu.edu.cn (c) The Author(s), 2020. Published by Cambridge University Press. This is an Open Access article, distributed under the terms of the Creative Commons Attribution licence (http://creativecommons.org/licenses/by/4.0/), which permits unrestricted re-use, distribution, and reproduction in any medium, provided the original work is properly cited.

\section{CAMBRIDGE} UNIVERSITY PRESS

\title{
Uptake of referrals for women with positive perinatal depression screening results and the effectiveness of interventions to increase uptake: a systematic review and meta-analysis
}

\section{WQ Xue ${ }^{1}, K_{K}$ Cheng $^{2}$, D Xu ${ }^{3}$ (D) X Jin ${ }^{1}$ and WJ Gong ${ }^{1}$ (D)}

${ }^{1}$ Xiangya School of Public Health, Central South University, Changsha, China; ${ }^{2}$ Institute of Applied Health Research, University of Birmingham, Birmingham, UK and ${ }^{3}$ Sun Yat-sen Global Health Institute, School of Public Health and Institute of State Governance, Sun Yat-sen University, Guangzhou, China

\begin{abstract}
Aims. Perinatal depression threatens the health of maternal women and their offspring. Although screening programs for perinatal depression exist, non-uptake of referral to further mental health care after screening reduces the utility of these programs. Uptake rates among women with positive screening varied widely across studies and little is known about how to improve the uptake rate. This study aimed to systematically review the available evidence on uptake rates, estimate the pooled rate, identify interventions to improve uptake of referral and explore the effectiveness of those interventions.

Methods. This systematic review has been registered in PROSPERO (registration number: CRD42019138095). We searched Pubmed, Web of Science, Cochrane Library, Ovid, Embase, CNKI, Wanfang Database and VIP Databases from database inception to January 13, 2019 and scanned reference lists of relevant researches for studies published in English or Chinese. Studies providing information on uptake rate and/or effectiveness of interventions on uptake of referral were eligible for inclusion. Studies were excluded if they did not report the details of the referral process or did not provide exact uptake rate. Data provided by observational studies and quasi-experimental studies were used to estimate the pooled uptake rate through meta-analysis. We also performed meta-regression and subgroup analyses to explore the potential source of heterogeneity. To evaluate the effectiveness of interventions, we conducted descriptive analyses instead of meta-analyses since there was only one randomised controlled trial (RCT).

Results. Of 2302 records identified, 41 studies were eligible for inclusion, including 39 observational studies $(n=9337)$, one quasi-experimental study $(n=43)$ and one RCT $(n=555)$. All but two studies were conducted in high-income countries. The uptake rates reported by included studies varied widely and the pooled uptake rate of referral was $43 \%$ (95\% confidence intervals [CI] 35-50\%) by a random-effect model. Meta-regression and subgroup analyses both showed that referral to on-site assessment or treatment $(60 \%, 95 \%$ CI $51-69 \%)$ had a significantly higher uptake rate than referral to mental health service $(32 \%, 95 \%$ CI $23-41 \%$ ) (odds ratio $1.31,95 \%$ CI 1.13-1.52). The included RCT showed that the referral intervention significantly improved the uptake rate $(p<0.01)$.

Conclusions. Almost three-fifths of women with positive screening results do not take up the referral offers after perinatal depression screening. Referral to on-site assessment and treatment may improve uptake of referral, but the quality of evidence on interventions to increase uptake was weak. More robust studies are needed, especially in low-and middle-income countries.
\end{abstract}

\section{Introduction}

Perinatal depression (PND) refers to depressive episodes that occur during pregnancy or after delivery. Affecting approximately $18 \%$ of all pregnancies worldwide (Gavin et al., 2005), this disorder is associated with many poor outcomes for women's maternal and physical health and the emotional and cognitive development of infants and children during their sensitive growth periods (Beardslee et al., 1998; Muzik and Borovska, 2010; Goodman et al., 2011). The early identification and timely intervention could improve the prognosis of PND means that in many countries screening for PND is incorporated as part of the routine perinatal care (Buist et al., 2008; Earls et al., 2010; Milgrom and Gemmill, 2014; O'Connor et al., 2016; Urato, 2017; ACOG, 2018). For screening to work, however, identification of women with positive screening results needs to be followed by timely intervention after referral. In this review, referral was defined as the process of recommending women to receive further mental health care after screening. The process of referral could be divided into three steps. First, the providers make the referral for women with positive screening results (step 1). 
Then, women accept the referral and try to access mental health services (step 2: 'uptake'). Finally, relevant resources must be available to provide the necessary support (step 3). Steps 1 and 3 are both responsibilities of the healthcare system and routine screening should only be carried out if the prerequisites of these two are met. This review, therefore, focuses on step 2, namely uptake by women with positive screening results after being offered referrals. Low uptake rates reduce the overall effectiveness of screening. If a woman does not take up the offer of referral after the positive screening, the basis of introducing universal screening is weakened (Hewitt and Gilbody, 2009; Thombs et al., 2014).

Previous studies investigating the uptake of referrals after screening have reported that the uptake rates varied very widely (0\% to 94\%) (Tam et al., 2002; Miller et al., 2009). Uptake of referral after depression screening is a complex process. There are barriers related to patients and healthcare providers. These needed to be addressed in order to increase the uptake rate. A systematic review of uptake would help to inform the debate on the case for screening in those countries where screening is not yet routinely performed. In those where screening is already taking place, understanding the reasons behind low uptake rates and what interventions would increase these rates would be useful to improve the effectiveness of screening.

In this paper, we presented a systematic review that included studies reporting uptake rates after screening for PND and a meta-analysis on the overall uptake rate. We also explored the relationship between the type of referral interventions after administering the screening test and uptake. In addition, we summarised the reasons behind the lack of engagement with mental health care after referral. In contrast to related reviews (Byatt et al., 2015; Long et al., 2019), we have specifically focused on uptake of referral (step 2) for reasons stated above.

\section{Methods}

\section{Literature review}

This systematic review and meta-analysis was undertaken according to Preferred Reporting Items for Systematic Reviews and Meta-Analyses (PRISMA) guidelines. The protocol was registered in the PROSPERO database, number CRD42019138095. One of us who has experiences in women's mental health (WG) developed the search strategy and the full search strategy is available in Appendix A. In brief, we searched Pubmed, Web of Science, Cochrane Library, Ovid, Embase, CNKI, Wanfang Database and VIP Database for studies in English or Chinese from the inception of the database until January 13, 2019. In English database, we used the search terms ('perinatal', 'pregnant', 'pregnancy', 'prenatal', 'antenatal', 'postnatal' OR 'postpartum') AND ('depression' OR 'depressive symptoms') AND 'screening' AND ('referral', 'referrals', 'refer', 'transfer' OR 'uptake'). We used the search terms (“围产期”, “孕期”, “产前” OR “产后”) AND (“抑郁” OR ‘抑 郁症”) AND “篮查” AND (“转诊” OR “转介’) in Chinese database.

\section{Inclusion and exclusion}

After removing duplications, we reviewed each title and abstract based on inclusion and exclusion criteria. Inclusion criteria were: (1) language limited to English and Chinese; (2) participants were pregnant or postpartum (within 2 years of delivery) women who were screened positive for PND by any validated screening tool; (3) reported uptake rates (the number of women who accept the referral and try to access mental health service among women who were offered referral); (4) if there were interventions other than the administering of the screening test, the objective or one of the objectives of the interventions was to improve referral status. Exclusion criteria were: (1) no detail of referral process or exact data of uptake of referral were reported; (2) case report or case series. Then full-text articles were retrieved to determine eligibility criteria. Finally, references of retrieved full-text articles were screened for additional eligible publications. Investigators (WX and LL/QL/JW/PY/XM) independently assessed each study for inclusion. Disagreements were resolved through discussion with each other or consulting a third one (WG). When the full-text was not available (e.g. only the abstract was available), we would contact the author by email and if no reply was received within a month, the article would be excluded.

\section{Extraction}

The following study-level characteristics were independently extracted (WX and LL/QL/JW/PY/XM) and disagreements were resolved via discussion or consulting a third one (WG): first author, publication year, study type, study country (countries are classified by income level according to World Bank Country and Lending Groups) (The World Bank Group, 2016), screening tool, sample size (number of women who were screened positive and were offered referral), time points of screening (prenatal, postnatal or perinatal), year of study, referral methods (referral to mental health services or on-site assessment or treatment), referral interventions, uptake of referral (number of women who accepted referrals and tried to access mental health service), referral uptake rates, the reasons for non-uptake and patient outcomes.

\section{Quality assessment}

We used the Loney criteria to assess the quality of observational studies and quasi-experimental studies, and used Cochrane Risk of Bias Tool (ROS) to assess the quality of randomised controlled trials (RCTs) (Loney et al., 1998; Higgins et al., 2011). The Loney criteria included eight items on the risk of bias from three aspects: A. Validity of the study methods: (1) The study design and sampling method are appropriate for the research question. (2) The sampling frame is appropriate. (3) The sample size is adequate. (4) Objective, suitable and standard criteria are used for the measurement of the health outcome. (5) The health outcome is measured in an unbiased fashion. (6) The response rate is adequate and the refusers are described. B. Interpretation of the results: (7) The estimates of prevalence and incidence are given with confidence intervals and in detail by subgroup, if appropriate. C. Applicability of the findings: (8) The study subjects and the setting are described in detail. The maximal total score is eight points, with higher scores suggesting a lower risk of bias. ROS helps to evaluate the risk of bias from six aspects: selection bias, performance bias, detection bias, attrition bias, reporting bias and others. Each item was determined as 'high risk of bias', 'low risk of bias', 'unclear risk of bias'. Assessment of bias was performed by two authors (WX and XJ) and disagreements were reconciled through discussion.

\section{Data analysis}

In our review, prospective or retrospective studies that did not assign women to intervention or control groups at the referral 
stage were considered observational studies. Before and after comparison studies which examined the difference before and after the referral intervention took place were regarded as quasi-experimental studies. Studies that randomly allocated women to a referral intervention group or no intervention group, or to a high-intensive referral intervention group or low-intensive referral intervention group were categorised as RCT. The data provided by observational studies and quasiexperimental studies were used to estimate the pooled rate of uptake. RCT was used to evaluate the effect of interventions.

The 'meta' module in R-3.5.1 statistical software package was used for the calculation of the pooled rate of uptake. First, the uptake rates reported in each study were transformed using the Freeman-Tukey Double arcsine method according to the distribution of rates (Freeman and Turkey, 1950; Luo et al., 2013). Then we calculated the pooled rate and $95 \%$ confidence intervals (CIs) in a meta-analysis. Heterogeneity was assessed using Cochran's Q test, and quantified by the $\mathrm{I}^{2}$ value and tau ${ }^{2}$. If the heterogeneity results showed that $p \leqslant 0.10$ or $I^{2}>50 \%$ and suggested high heterogeneity, the random-effect model would be adopted. Otherwise, the fixed-effect model would be applied. Publication bias was evaluated by presenting a funnel plot and performing Egger's linear test. Sensitivity analysis was performed by serially removing studies one by one to explore the impact of doing so on the overall uptake rate. Any study for which removal substantially changed the uptake rate would be noted. Multivariate meta-regression was conducted to explore the source of heterogeneity and then the odds ratios (OR) were calculated through a formula: $\mathrm{OR}=\exp (\beta)(\beta$ : regression coefficient). Through literature review, study country, time points of screening, referral methods and referral interventions were identified as predictor variables (Smith et al., 2009; Byatt et al., 2015; Gajaria and Ravindran, 2018; Savovic et al., 2018). Subgroup analyses were performed to estimate the pooled uptake rate with regard to the significant factors in meta-regression analyses and the differences between subgroups were investigated through chi-squared test (the significance level was $p<0.05$ ).

There were insufficient numbers of RCTs to allow for meta-analysis. Therefore, we conducted a descriptive analysis based on the uptake rates provided by the RCT in SPSS 18.0 to show the effectiveness of interventions.

\section{Results}

\section{Search results}

The systematic literature search yielded 2302 articles, including 2296 English articles and six Chinese articles (Fig. 1). After removing duplicates, a total of 1818 references were identified. Of the 1818 reviewed, 1681 were eliminated after title/abstract review and 105 were eliminated after full-text reviews because they did not meet inclusion criteria (The main reasons for exclusion are shown in Fig. 1.) This resulted in 32 studies for inclusion. After additional searches, a further nine studies were included from reference lists of included papers. In total, 41 articles were included in this review, including one RCT, one before and after comparison study, and 39 observational studies.

\section{Quality assessment}

Consensus was reached in over $90 \%$ among the two independent authors (WX and XJ) in quality assessment. In 40 observational

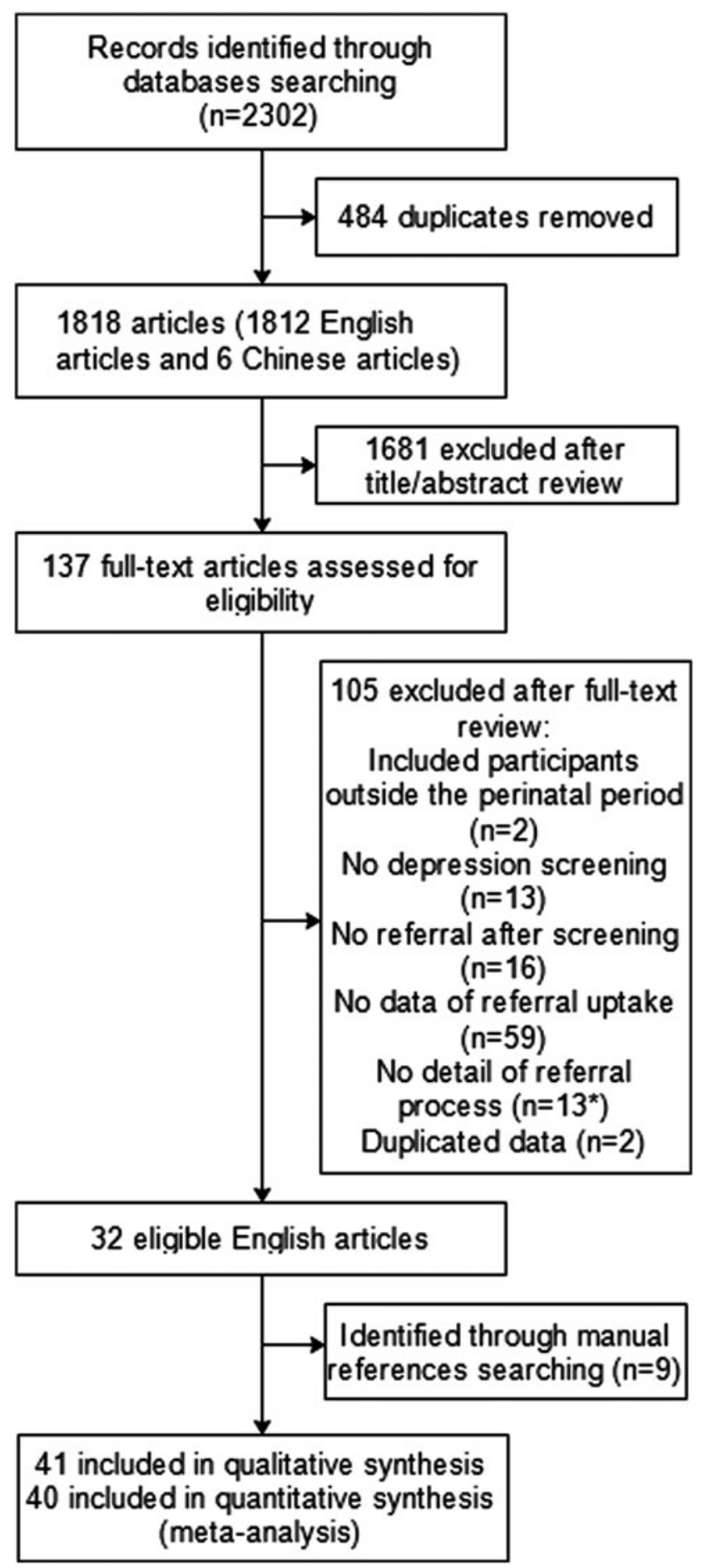

Fig. 1. Article selection process. *Three articles were excluded because we did not get reply within 1 month after we contacted the authors by email.

or quasi-experimental studies, 12 of the 40 eligible studies scored five points, 15 scored four points, 11 scored three points and two scored two points based on the Loney criteria. For the validity of the study methods, $62 \%$ of studies $(n=25)$ used a biased sampling method, $95 \%$ of the studies $(n=38)$ did not apply an appropriate sampling frame, $72 \%(n=29)$ had an insufficient sample size and $72 \%(n=29)$ had low response rates or did not describe refusers. None provided confidence intervals. Three studies did not report 
the characteristics of study subjects. Result of the quality assessment of the RCT is shown in Appendix B. The RCT had 'high risk' in performance bias which meant it did not blind the randomisation status to participants or personnel.

\section{Study characteristics}

Table 1 shows the characteristics of the eligible studies. Forty studies were observational or quasi-experimental studies with 9380 women who were screen positive. One was RCT with 555 women who were screen positive. Two-thirds of the 41 studies $(n=27)$ were carried out in the USA. The rest were conducted in Australia $(n=6)$, Iceland $(n=2)$ and there was one study each from Turkey, Singapore, New Zealand, China, Republic of South Africa and Israel. The sample size ranged from 5 to 1751 participants. Mean sample ages of women ranged from 23.4 to 35.7 years.

Most studies $(n=38,93 \%)$ used the Edinburgh Postnatal Depression Scale (EPDS) as the screening tool (Cox et al., 1987). The remaining used Patient Health Questionnaire (PHQ-9) ( $n=2)$ (Wittkampf et al., 2007), Beck Depression Inventory (BDI) $(n=1)$ (Beck et al., 1961), Behavioural Health Risk Screen (BHRS) $(n=1)$ (Price et al., 2012), self-assessment of Depression, General Scale (SAD-G) $(n=1)$ (Snaith et al., 1976) and Depression Anxiety Stress Scales (DASS) $(n=1)$ (Lovibond and Lovibond, 1995). Time points of screening ranged from first antenatal checks to 2 years after delivery, which was broadly in line with the timing of prenatal/postnatal appointments or well-baby visits. Main screening sites were healthcare facilities, including obstetrics, paediatrics or neonatal intensive care units. After identifying women who were screened positive, programme members or health care providers who performed screening, including obstetricians, nurses, or midwives, would recommend referral. Some studies conducted remote screening through mail or telephone. The majority of studies referred women with positive screening results to mental health service for further mental health assessment or treatment. Providers of mental health services included mental health professionals (e.g. psychologists, psychiatrists), perinatal care providers (e.g. obstetricians, midwives), primary care providers (e.g. general practitioner) and behavioural health specialists. Some studies provided on-site assessment or treatment, which were mainly performed in the clinic by program members, social workers, or health care providers who conducted the screening. When women who were screened positive attended mental health department/clinic to undergo further assessment/treatment or received on-site assessment/treatment provided by studies, it constituted as uptake of referral in this review. In prospective studies, the outcomes of referral were mostly determined through follow-up by telephone. Of the prospective studies, 12 provided the length of follow-up which ranged from 2 weeks to 6 months. In retrospective studies, the outcomes of referral were always determined by reviewing the medical records.

Out of the 41 studies included in this review, 10 of them reported patient outcomes related to their depression (Appendix C). All ten studies showed improvement among patients who received further specialist support after referral.

\section{Pooled referral uptake rate}

In 40 observational or quasi-experimental studies, three provided referral data of both prenatal and postnatal periods, one provided data of pre-intervention and post-intervention. Therefore, we had a total of 44 referral rates from 40 observational or quasiexperimental studies for the following analysis. The rates of referral uptake in different countries varied widely as Table 1 shows.

Figure 2 shows the uptake rates of all included observational or quasi-experimental studies and the pooled rate. Significant heterogeneity $\left(I^{2}=97.7 \%, \tau^{2}=0.0537, p<0.01\right)$ was observed across the included studies. The pooled uptake rate was $43 \%$ (95\% CI 35-50\%), using the random effect model.

\section{Publication bias and sensitivity analysis}

The funnel plot was almost symmetrical suggesting that publication bias was likely to be small (see Appendix D). This was consistent with the result of the Egger's test $(t=-0.32, p=0.75)$.

Sensitivity analysis was performed through serially removing studies one by one. The $\mathrm{I}^{2}$ values ranged from $97.3 \%$ to $97.8 \%$, the $\mathrm{tau}^{2}$ ranged from 0.0477 to 0.0655 and the combined referral rate ranged from $41 \%(95 \%$ CI $34-48 \%$ ) to $44 \%$ (95\% CI $37-$ $52 \%)$, indicating that the results of the meta-analysis were reliable.

\section{Meta-regression analysis}

The results of multivariate regression analysis are provided in Table 2. Referral methods were associated with the rate of uptake of referral but not with study country, time points of screening and referral interventions. The uptake rate where on-site assessment or treatment was used was significantly higher than the rate where the intervention was a referral to the mental health sector $(\mathrm{OR}=1.31,95 \% \mathrm{CI} 1.13-1.52)$.

\section{Subgroup analyses}

Subgroup analyses by referral methods showed significant differences in the uptake rate $\left(\chi^{2}=17.95, p<0.01\right)$. Studies that provided on-site assessment or treatment had higher rates $(60 \%$, 95\% CI 51-69\%) than studies that referred women to mental health service (32\%, 95\% CI 23-41\%).

\section{Referral interventions for women who were screened positive}

Table 3 shows the referral interventions in eligible studies. Table 4 shows the characteristics of the RCT, which showed a significant difference in uptake rates between intervention and control group.

\section{The reasons for non-uptake among screen-positive women}

We examined the reasons for non-uptake at women or provider levels. For the former, the most frequent reasons were lack of time' and 'perception that mood had improved'. Others cited 'cost concerns' or 'transportation problems'. The perception of the nature of PND also affected referrals. Some declined referral because of stigma associated with psychiatric treatments. Some thought 'it is normal to have some depression in the puerperium' and therefore did not perceive the need for further health care. Women's preferences for the type of service offered also influenced the level of acceptance. It was reported that women were 'not interested in receiving specialised services; home visitation was generally a much more acceptable referral' and some women tended to 'use their own resources' instead of resorting to support provided by the research team. The reasons related to health care provider level mainly concerned the interaction between women with positive screening results and health care providers. Some women 
Table 1. Characteristics of studies included in this review

\begin{tabular}{|c|c|c|c|c|c|c|c|}
\hline Author (year) & $\begin{array}{l}\text { Study type, country and year } \\
\text { of study }\end{array}$ & $\begin{array}{l}\text { Screening tool and time } \\
\text { points }\end{array}$ & Referral methods & Referral interventions & $\begin{array}{l}\text { Sample size and } \\
\text { uptake of referral }\end{array}$ & $\begin{array}{l}\text { Uptake } \\
\text { rate }\end{array}$ & $\begin{array}{l}\text { Quality } \\
\text { score }\end{array}$ \\
\hline Lydsdottir et al. (2019) & $\begin{array}{l}\text { Observational study; Iceland; } \\
\text { 2006-2011 }\end{array}$ & $\begin{array}{l}\text { EPDS, DASS; } 16 \text { weeks } \\
\text { gestation }\end{array}$ & $\begin{array}{l}\text { Referral to mental } \\
\text { health service }\end{array}$ & $\begin{array}{l}\text { Provision of mental health } \\
\text { consultation or diagnosis }\end{array}$ & $396 ; 273$ & $69 \%$ & 4 \\
\hline Kallem et al. (2019) & $\begin{array}{l}\text { Observational study; USA; } \\
\text { 2011-2014 }\end{array}$ & EPDS; 2-month postnatal & $\begin{array}{l}\text { Referral to mental } \\
\text { health service }\end{array}$ & $\begin{array}{l}\text { Provision of resources, assistance in } \\
\text { referral }\end{array}$ & $195 ; 23$ & $12 \%$ & 4 \\
\hline Jarvis et al. (2018) & $\begin{array}{l}\text { Observational study; USA; } \\
\text { 2015-2016 }\end{array}$ & $\begin{array}{l}\text { EPDS; within } 6 \text { months } \\
\text { postnatal }\end{array}$ & $\begin{array}{l}\text { Referral to mental } \\
\text { health service }\end{array}$ & $\begin{array}{l}\text { Education, provision of resources, } \\
\text { provision of mental health } \\
\text { consultation or diagnosis, supportive } \\
\text { treatment or referral to support } \\
\text { group }\end{array}$ & $37 ; 6$ & $16 \%$ & 3 \\
\hline Bauer et al. (2017) & $\begin{array}{l}\text { Observational study; USA; } \\
\text { 2012-2014 }\end{array}$ & $\begin{array}{l}\text { EPDS; within } 15 \text { months } \\
\text { postnatal }\end{array}$ & $\begin{array}{l}\text { Referral to mental } \\
\text { health service }\end{array}$ & Education, provision of resources & $73 ; 36$ & $49 \%$ & 3 \\
\hline Price et al. (2017) & Observational study; USA; / & BHRS; perinatal & $\begin{array}{l}\text { Referral to mental } \\
\text { health service }\end{array}$ & I & $330 ; 92$ & $28 \%$ & 5 \\
\hline \multirow[t]{2}{*}{ Venkatesh et al. (2016) } & \multirow{2}{*}{$\begin{array}{l}\text { Observational study; USA; } \\
\text { 2010-2014 }\end{array}$} & EPDS; $24-28$ weeks gestation & \multirow{2}{*}{$\begin{array}{l}\text { On-site assessment } \\
\text { or treatment }\end{array}$} & \multirow{2}{*}{$\begin{array}{l}\text { On-site assessment, assistance in } \\
\text { referral }\end{array}$} & $396 ; 327$ & $83 \%$ & \multirow[t]{2}{*}{5} \\
\hline & & EPDS; 6-week postnatal & & & $180 ; 128$ & $71 \%$ & \\
\hline Mestad et al. (2016) & $\begin{array}{l}\text { Observational study; USA; } \\
2010\end{array}$ & $\begin{array}{l}\text { EPDS; first prenatal visit and } \\
\text { at 26-week of pregnancy }\end{array}$ & $\begin{array}{l}\text { On-site assessment } \\
\text { or treatment }\end{array}$ & On-site assessment & $55 ; 35$ & $64 \%$ & 4 \\
\hline BenDavid et al. (2016) & $\begin{array}{l}\text { Observational study; USA; } \\
2013\end{array}$ & EPDS; 2-3 weeks postnatal & $\begin{array}{l}\text { Referral to mental } \\
\text { health service }\end{array}$ & $\begin{array}{l}\text { Education, provision of resources, } \\
\text { assistance in referral, health care } \\
\text { provider training }\end{array}$ & $14 ; 8$ & $57 \%$ & 5 \\
\hline Trost et al. (2016) & $\begin{array}{l}\text { Observational study; USA; } \\
\text { 2013-2014 }\end{array}$ & $\begin{array}{l}\text { EPDS; } 2 \text { weeks to } 1 \text { year } \\
\text { postnatal }\end{array}$ & $\begin{array}{l}\text { Referral to mental } \\
\text { health service }\end{array}$ & $\begin{array}{l}\text { Education, provision of resources, } \\
\text { provision of mental health } \\
\text { consultation or diagnosis, health care } \\
\text { provider training }\end{array}$ & $21 ; 8$ & $38 \%$ & 3 \\
\hline Boyd et al. (2015) & Observational study; USA; / & EPDS; perinatal & $\begin{array}{l}\text { Referral to mental } \\
\text { health service }\end{array}$ & Education, assistance in referral & $38 ; 21$ & $55 \%$ & 3 \\
\hline Bina (2014) & $\begin{array}{l}\text { Observational study; Israel; } \\
\text { 2008-2009 }\end{array}$ & EPDS; 6-week postnatal & $\begin{array}{l}\text { Referral to mental } \\
\text { health service }\end{array}$ & Education & $88 ; 21$ & $24 \%$ & 4 \\
\hline Emerson et al. (2014) & $\begin{array}{l}\text { Observational study; USA; } \\
\text { 2011-2012 }\end{array}$ & $\begin{array}{l}\text { EPDS; within } 4 \text { months } \\
\text { postnatal }\end{array}$ & $\begin{array}{l}\text { Referral to mental } \\
\text { health service }\end{array}$ & $\begin{array}{l}\text { Provision of resources, provision of } \\
\text { mental health consultation or } \\
\text { diagnosis }\end{array}$ & $12 ; 2$ & $17 \%$ & 3 \\
\hline Nelson et al. (2013) & $\begin{array}{l}\text { Observational study; USA; } \\
\text { 2008-2010 }\end{array}$ & EPDS; first postnatal visit & $\begin{array}{l}\text { Referral to mental } \\
\text { health service }\end{array}$ & Assistance in referral & $1106 ; 250$ & $23 \%$ & 5 \\
\hline Stock et al. (2013) & $\begin{array}{l}\text { Observational study; } \\
\text { Australia; / }\end{array}$ & $\begin{array}{l}\text { EPDS; } 2 \text { weeks to } 6 \text { months } \\
\text { postnatal }\end{array}$ & $\begin{array}{l}\text { On-site assessment } \\
\text { or treatment }\end{array}$ & $\begin{array}{l}\text { On-site assessment, provision of } \\
\text { resources }\end{array}$ & $39 ; 27$ & $69 \%$ & 4 \\
\hline Wisner et al. (2013) & $\begin{array}{l}\text { Observational study; USA; } \\
\text { 2006-2010 }\end{array}$ & EPDS; 4-6 weeks postnatal & $\begin{array}{l}\text { On-site assessment } \\
\text { or treatment }\end{array}$ & $\begin{array}{l}\text { On-site assessment, education, } \\
\text { health care provider training }\end{array}$ & $1396 ; 826$ & $59 \%$ & 4 \\
\hline \multirow[t]{2}{*}{ Rowan et al. (2012) } & \multirow{2}{*}{$\begin{array}{l}\text { Observational study; USA; } \\
\text { 2008-2009 }\end{array}$} & EPDS; first prenatal visit & \multirow{2}{*}{$\begin{array}{l}\text { Referral to mental } \\
\text { health service }\end{array}$} & \multirow[t]{2}{*}{ Assistance in referral } & $102 ; 0$ & $0 \%$ & \multirow[t]{2}{*}{4} \\
\hline & & EPDS; 6-week postnatal & & & $28 ; 5$ & $18 \%$ & \\
\hline
\end{tabular}


Table 1. (Continued.)

\begin{tabular}{|c|c|c|c|c|c|c|c|}
\hline Author (year) & $\begin{array}{l}\text { Study type, country and year } \\
\text { of study }\end{array}$ & $\begin{array}{l}\text { Screening tool and time } \\
\text { points }\end{array}$ & Referral methods & Referral interventions & $\begin{array}{l}\text { Sample size and } \\
\text { uptake of referral }\end{array}$ & $\begin{array}{l}\text { Uptake } \\
\text { rate }\end{array}$ & $\begin{array}{l}\text { Quality } \\
\text { score }\end{array}$ \\
\hline Segre et al. (2012) & $\begin{array}{l}\text { Observational study; USA; } \\
\text { 2002-2009 }\end{array}$ & $\begin{array}{l}\text { EPDS; prenatal to } 2 \text { years } \\
\text { postnatal }\end{array}$ & $\begin{array}{l}\text { On-site assessment } \\
\text { or treatment }\end{array}$ & $\begin{array}{l}\text { Provision of mental health } \\
\text { consultation or diagnosis, supportive } \\
\text { treatment or referral to support } \\
\text { group, health care provider training, } \\
\text { on-site assessment or treatment }\end{array}$ & $573 ; 271$ & $47 \%$ & 5 \\
\hline Honikman et al. (2012) & $\begin{array}{l}\text { Observational study; South } \\
\text { Africa; 2008-2011 }\end{array}$ & EPDS; perinatal & $\begin{array}{l}\text { On-site assessment } \\
\text { or treatment }\end{array}$ & $\begin{array}{l}\text { On-site assessment, health care } \\
\text { provider training }\end{array}$ & $1751 ; 832$ & $48 \%$ & 5 \\
\hline \multirow[t]{2}{*}{ Miller et al. (2012) } & \multirow[t]{2}{*}{$\begin{array}{l}\text { Before and after comparison; } \\
\text { USA; 2008-2009 }\end{array}$} & \multirow[t]{2}{*}{ PHQ-9; postnatal } & \multirow[t]{2}{*}{$\begin{array}{l}\text { On-site assessment } \\
\text { or treatment }\end{array}$} & $\begin{array}{l}\text { On-site assessment (by behavioural } \\
\text { health specialists) }\end{array}$ & $10 ; 1$ & $10 \%$ & \multirow[t]{2}{*}{4} \\
\hline & & & & $\begin{array}{l}\text { On-site assessment (by perinatal care } \\
\text { providers), health care provider } \\
\text { training }\end{array}$ & $33 ; 28$ & $85 \%$ & \\
\hline \multirow[t]{2}{*}{ Yawn et al. (2012) } & \multirow[t]{2}{*}{$\begin{array}{l}\text { Cluster RCTs; USA; 2006- } \\
2010\end{array}$} & \multirow[t]{2}{*}{$\begin{array}{l}\text { EPDS, PHQ-9; 5-12 weeks } \\
\text { postnatal }\end{array}$} & $\begin{array}{l}\text { On-site assessment } \\
\text { or treatment }\end{array}$ & $\begin{array}{l}\text { On-site assessment, health care } \\
\text { provider training, provision of tools } \\
\text { to facilitate the management of PND }\end{array}$ & $322 ; 194$ & $60 \%$ & \multirow[t]{2}{*}{ l } \\
\hline & & & $\begin{array}{l}\text { Referral to mental } \\
\text { health service }\end{array}$ & Short health care provider training & $233 ; 78$ & $33 \%$ & \\
\hline Milgrom et al. (2011) & $\begin{array}{l}\text { Observational study; } \\
\text { Australia; / }\end{array}$ & $\begin{array}{l}\text { EPDS; } 6 \text { weeks- } 4 \text { months } \\
\text { postnatal }\end{array}$ & $\begin{array}{l}\text { Referral to mental } \\
\text { health service }\end{array}$ & $\begin{array}{l}\text { Health care provider training, } \\
\text { supportive treatment or referral to } \\
\text { support group }\end{array}$ & $333 ; 68$ & $20 \%$ & 4 \\
\hline \multirow[t]{2}{*}{ Burton et al. (2011) } & \multirow{2}{*}{$\begin{array}{l}\text { Observational study; USA; } \\
2006\end{array}$} & EPDS; 36-week gestation & \multirow{2}{*}{$\begin{array}{l}\text { Referral to mental } \\
\text { health service }\end{array}$} & \multirow{2}{*}{$\begin{array}{l}\text { Provision of mental health } \\
\text { consultation or diagnosis }\end{array}$} & $3 ; 1$ & $33 \%$ & \multirow[t]{2}{*}{5} \\
\hline & & EPDS; 6 weeks postnatal & & & $34 ; 17$ & $50 \%$ & \\
\hline Chen et al. (2011) & $\begin{array}{l}\text { Observational study; } \\
\text { Singapore; 2008-2009 }\end{array}$ & $\begin{array}{l}\text { EPDS; } 2 \text { weeks to } 6 \text { months } \\
\text { postnatal }\end{array}$ & $\begin{array}{l}\text { On-site assessment } \\
\text { or treatment }\end{array}$ & $\begin{array}{l}\text { Education, supportive treatment or } \\
\text { referral to support group, on-site } \\
\text { assessment or treatment }\end{array}$ & $126 ; 41$ & $33 \%$ & 3 \\
\hline Reay et al. (2011) & $\begin{array}{l}\text { Observational study; } \\
\text { Australia; } 2004\end{array}$ & $\begin{array}{l}\text { EPDS; prenatal and } 6-8 \text { weeks } \\
\text { postnatal }\end{array}$ & $\begin{array}{l}\text { Referral to mental } \\
\text { health service }\end{array}$ & $\begin{array}{l}\text { Provision of resources, assistance in } \\
\text { referral }\end{array}$ & $98 ; 62$ & $63 \%$ & 4 \\
\hline Kim et al. (2010) & $\begin{array}{l}\text { Observational study; USA; } \\
\text { 2006-2007 }\end{array}$ & EPDS; perinatal & $\begin{array}{l}\text { Referral to mental } \\
\text { health service }\end{array}$ & l & $28 ; 4$ & $14 \%$ & 3 \\
\hline Leung et al. (2011) & $\begin{array}{l}\text { Observational study; Hong } \\
\text { Kong, China; 2005-2006 }\end{array}$ & EPDS; 2-month postnatal & $\begin{array}{l}\text { On-site assessment } \\
\text { or treatment }\end{array}$ & On-site assessment & $67 ; 51$ & $76 \%$ & 5 \\
\hline Horowitz et al. (2009) & $\begin{array}{l}\text { Observational study; USA; } \\
\text { 2004-2007 }\end{array}$ & EPDS; 4-6 weeks postnatal & $\begin{array}{l}\text { Referral to mental } \\
\text { health service }\end{array}$ & $\begin{array}{l}\text { Provision of mental health } \\
\text { consultation or diagnosis, assistance } \\
\text { in referral }\end{array}$ & $674 ; 185$ & $27 \%$ & 4 \\
\hline Miller et al. (2009) & Observational study; USA; / & $\begin{array}{l}\text { EPDS; first prenatal visit, } \\
\text { 28-week gestation and } \\
\text { postnatal }\end{array}$ & $\begin{array}{l}\text { On-site assessment } \\
\text { or treatment }\end{array}$ & $\begin{array}{l}\text { On-site assessment, health care } \\
\text { provider training }\end{array}$ & $174 ; 164$ & $94 \%$ & 2 \\
\hline Orhon et al. (2007) & $\begin{array}{l}\text { Observational study; Turkey; } \\
\text { / }\end{array}$ & EPDS; within 1 year postnatal & $\begin{array}{l}\text { Referral to mental } \\
\text { health service }\end{array}$ & $\begin{array}{l}\text { Provision of mental health } \\
\text { consultation or diagnosis }\end{array}$ & $35 ; 28$ & $80 \%$ & 3 \\
\hline Harvey and Pun (2007) & $\begin{array}{l}\text { Observational study; } \\
\text { Australia; 2003-2005 }\end{array}$ & EPDS; prenatal & $\begin{array}{l}\text { Referral to mental } \\
\text { health service }\end{array}$ & I & $102 ; 52$ & $51 \%$ & 4 \\
\hline
\end{tabular}




\begin{tabular}{|c|c|c|c|c|c|c|c|}
\hline $\begin{array}{l}\text { Ingadottir and Thome } \\
\text { (2006) }\end{array}$ & $\begin{array}{l}\text { Observational study; Iceland; } \\
\text { 2001-2002 }\end{array}$ & EPDS; 9-week postnatal & $\begin{array}{l}\text { On-site assessment } \\
\text { or treatment }\end{array}$ & $\begin{array}{l}\text { On-site assessment or treatment, } \\
\text { supportive treatment or referral to } \\
\text { support group }\end{array}$ & $32 ; 22$ & $69 \%$ & 5 \\
\hline Gordon et al. (2006) & $\begin{array}{l}\text { Observational study; USA; } \\
2003-2005\end{array}$ & $\begin{array}{l}\text { EPDS; } 28-32 \text { weeks of } \\
\text { gestation, 6-week postnatal }\end{array}$ & $\begin{array}{l}\text { Referral to mental } \\
\text { health service }\end{array}$ & $\begin{array}{l}\text { Provision of mental health } \\
\text { consultation or diagnosis, assistance } \\
\text { in referral, health care provider } \\
\text { training }\end{array}$ & $487 ; 303$ & $62 \%$ & 5 \\
\hline $\begin{array}{l}\text { Horowitz and Cousins } \\
(2006)\end{array}$ & Observational study; USA; / & EPDS; 2-4 weeks postnatal & $\begin{array}{l}\text { Referral to mental } \\
\text { health service }\end{array}$ & Assistance in referral & $117 ; 21$ & $18 \%$ & 4 \\
\hline Flynn et al. (2006) & $\begin{array}{l}\text { Observational study; USA; } \\
\text { 2002-2004 }\end{array}$ & EPDS; first prenatal visit & $\begin{array}{l}\text { On-site assessment } \\
\text { or treatment }\end{array}$ & $\begin{array}{l}\text { Education, on-site assessment or } \\
\text { treatment, assistance in referral }\end{array}$ & $73 ; 19$ & $26 \%$ & 4 \\
\hline Carter et al. (2005) & $\begin{array}{l}\text { Observational study; New } \\
\text { Zealand; / }\end{array}$ & EPDS; $12-22$ weeks gestation & $\begin{array}{l}\text { On-site assessment } \\
\text { or treatment }\end{array}$ & On-site assessment & $12 ; 7$ & $58 \%$ & 3 \\
\hline Chaudron et al. (2004) & $\begin{array}{l}\text { Observational study; USA; } \\
\text { 1998-2001 }\end{array}$ & EPDS; 1-year postnatal & $\begin{array}{l}\text { On-site assessment } \\
\text { or treatment }\end{array}$ & On-site assessment or treatment & $16 ; 7$ & $44 \%$ & 5 \\
\hline Miller et al. (2004) & $\begin{array}{l}\text { Observational study; USA; } \\
2003\end{array}$ & EPDS; 4-6 weeks postnatal & $\begin{array}{l}\text { Referral to mental } \\
\text { health service }\end{array}$ & $\begin{array}{l}\text { Education, provision of resources, } \\
\text { assistance in referral }\end{array}$ & $23 ; 5$ & $22 \%$ & 5 \\
\hline Tam et al. (2002) & Observational study; USA; / & $\begin{array}{l}\text { EPDS,BDI; } 6-8 \text { weeks, } 4,6,9,12 \\
\text { months postnatal }\end{array}$ & $\begin{array}{l}\text { Referral to mental } \\
\text { health service }\end{array}$ & $\begin{array}{l}\text { Provision of mental health } \\
\text { consultation or diagnosis }\end{array}$ & $5 ; 0$ & $0 \%$ & 2 \\
\hline Fergerson et al. (2002) & $\begin{array}{l}\text { Observational study; USA; } \\
2001\end{array}$ & EPDS; 4-6 weeks postnatal & $\begin{array}{l}\text { On-site assessment } \\
\text { or treatment }\end{array}$ & On-site assessment & $11 ; 5$ & $45 \%$ & 3 \\
\hline Rhonda et al. (1994) & $\begin{array}{l}\text { Observational study; } \\
\text { Australia; } 1989\end{array}$ & EPDS; 8-9 months postnatal & $\begin{array}{l}\text { Referral to mental } \\
\text { health service }\end{array}$ & l & $45 ; 6$ & $13 \%$ & 3 \\
\hline $\begin{array}{l}\text { Robinson and Young } \\
\text { (1982) }\end{array}$ & $\begin{array}{l}\text { Observational study; } \\
\text { Australia; } 1980\end{array}$ & SAD-G; 6-8 weeks postnatal & $\begin{array}{l}\text { Referral to mental } \\
\text { health service }\end{array}$ & $\begin{array}{l}\text { Provision of mental health } \\
\text { consultation or diagnosis }\end{array}$ & $12 ; 6$ & $50 \%$ & 4 \\
\hline
\end{tabular}




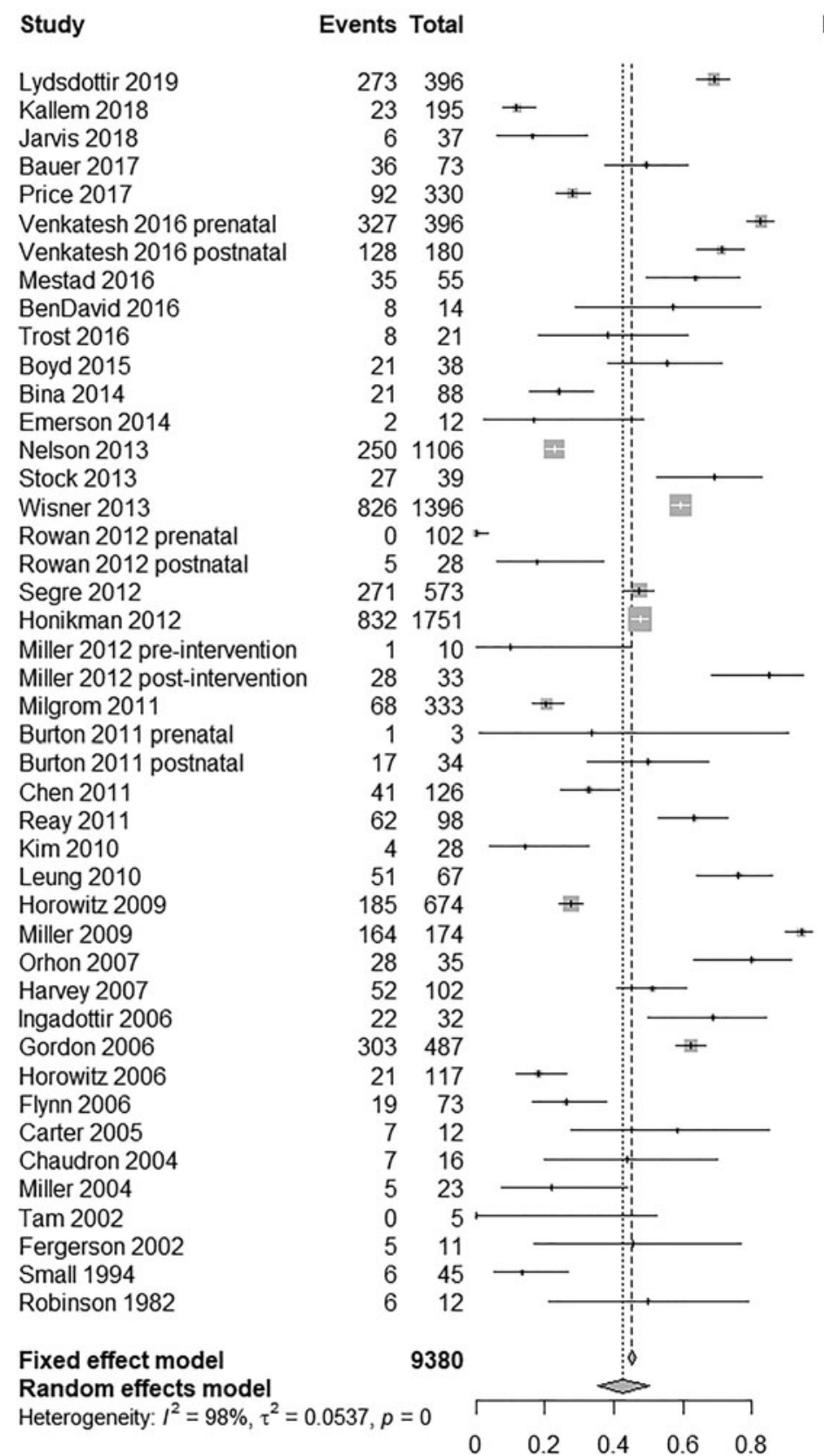

Proportion $\quad 95 \%-\mathrm{Cl} \quad \begin{array}{r}\text { Weight } \\ \text { (fixed) }\end{array} \begin{array}{r}\text { Weight } \\ \text { (random) }\end{array}$

\begin{tabular}{llrl}
0.69 & {$[0.64 ; 0.73]$} & $4.2 \%$ & $2.5 \%$ \\
$0.12[0.08 ; 0.17]$ & $2.1 \%$ & $2.5 \%$ \\
$0.16[0.06 ; 0.32]$ & $0.4 \%$ & $2.3 \%$ \\
$0.49[0.37 ; 0.61]$ & $0.8 \%$ & $2.4 \%$ \\
$0.28[0.23 ; 0.33]$ & $3.5 \%$ & $2.5 \%$ \\
$0.83[0.78 ; 0.86]$ & $4.2 \%$ & $2.5 \%$ \\
$0.71[0.64 ; 0.78]$ & $1.9 \%$ & $2.5 \%$ \\
$0.64[0.50 ; 0.76]$ & $0.6 \%$ & $2.4 \%$ \\
$0.57[0.29 ; 0.82]$ & $0.2 \%$ & $1.9 \%$ \\
$0.38[0.18 ; 0.62]$ & $0.2 \%$ & $2.1 \%$ \\
$0.55[0.38 ; 0.71]$ & $0.4 \%$ & $2.3 \%$ \\
$0.24[0.15 ; 0.34]$ & $0.9 \%$ & $2.4 \%$ \\
$0.17[0.02 ; 0.48]$ & $0.1 \%$ & $1.9 \%$ \\
$0.23[0.20 ; 0.25]$ & $11.8 \%$ & $2.6 \%$ \\
$0.69[0.52 ; 0.83]$ & $0.4 \%$ & $2.3 \%$ \\
$0.59[0.57 ; 0.62]$ & $14.9 \%$ & $2.6 \%$ \\
$0.00[0.00 ; 0.04]$ & $1.1 \%$ & $2.5 \%$ \\
$0.18[0.06 ; 0.37]$ & $0.3 \%$ & $2.2 \%$ \\
$0.47[0.43 ; 0.51]$ & $6.1 \%$ & $2.5 \%$ \\
$0.48[0.45 ; 0.50]$ & $18.6 \%$ & $2.6 \%$ \\
$0.10[0.00 ; 0.45]$ & $0.1 \%$ & $1.8 \%$ \\
$0.85[0.68 ; 0.95]$ & $0.4 \%$ & $2.3 \%$ \\
$0.20[0.16 ; 0.25]$ & $3.5 \%$ & $2.5 \%$ \\
$0.33[0.01 ; 0.91]$ & $0.0 \%$ & $1.1 \%$ \\
$0.50[0.32 ; 0.68]$ & $0.4 \%$ & $2.3 \%$ \\
$0.33[0.24 ; 0.41]$ & $1.3 \%$ & $2.5 \%$ \\
$0.63[0.53 ; 0.73]$ & $1.0 \%$ & $2.5 \%$ \\
$0.14[0.04 ; 0.33]$ & $0.3 \%$ & $2.2 \%$ \\
$0.76[0.64 ; 0.86]$ & $0.7 \%$ & $2.4 \%$ \\
$0.27[0.24 ; 0.31]$ & $7.2 \%$ & $2.6 \%$ \\
$0.94[0.90 ; 0.97]$ & $1.9 \%$ & $2.5 \%$ \\
$0.80[0.63 ; 0.92]$ & $0.4 \%$ & $2.3 \%$ \\
$0.51[0.41 ; 0.61]$ & $1.1 \%$ & $2.5 \%$ \\
$0.69[0.50 ; 0.84]$ & $0.3 \%$ & $2.2 \%$ \\
$0.62[0.58 ; 0.67]$ & $5.2 \%$ & $2.5 \%$ \\
$0.18[0.11 ; 0.26]$ & $1.2 \%$ & $2.5 \%$ \\
$0.26[0.16 ; 0.38]$ & $0.8 \%$ & $2.4 \%$ \\
$0.58[0.28 ; 0.85]$ & $0.1 \%$ & $1.9 \%$ \\
$0.44[0.20 ; 0.70]$ & $0.2 \%$ & $2.0 \%$ \\
$0.22[0.07 ; 0.44]$ & $0.2 \%$ & $2.1 \%$ \\
$0.00[0.00 ; 0.52]$ & $0.1 \%$ & $1.4 \%$ \\
$0.45[0.17 ; 0.77]$ & $0.1 \%$ & $1.8 \%$ \\
$0.13[0.05 ; 0.27]$ & $0.5 \%$ & $2.3 \%$ \\
$0.50[0.21 ; 0.79]$ & $0.1 \%$ & $1.9 \%$ \\
$0.45[0.44 ; 0.46]$ & $100.0 \%$ & \\
$0.43[0.35 ; 0.50]$ & & -- & $100.0 \%$ \\
\hline
\end{tabular}

Fig. 2. Forest plot of the results of the meta-analysis of referral uptake rates in included studies.

declined because of disagreement with their health care providers. For instance, some women wanted 'a quick fix' while psychiatrists 'looked for something long term like counseling'. Meanwhile, the attitude of providers could influence referrals. 'Women appreciated health professionals who gave their time, acknowledged their feelings and offered support'; and 'when women's feelings were denied, when they felt unable or were not given the opportunity to talk, then the experience of seeking help could be a very negative one'.

\section{Discussion}

\section{Principal findings}

Our systematic review and meta-analysis identified 41 studies that reported the uptake rate of referral and the effect of interventions for women with positive PND screening results. These included 39 observational studies and one quasi-experimental study (total $N=9380$ women with positive screening results) which were used to estimate the pooled uptake rate, and one RCT $(N=555$ women with positive screening results) which was used to evaluate the effectiveness of referral interventions. The overall pooled uptake rate for women with positive screening results was $43 \%$ (95\% CI 35-50\%). Where the women were referred to was the most important determinant. Studies that provided on-site assessment or treatment had higher uptake rates than studies that referred the women to a separate mental health service. The RCT showed significant improvement in uptake rates after the implementation of referral interventions. The more frequently mentioned reasons for refusing referral were 'lack of time' and 'perception that mood had improved'. 
Table 2. Multivariate meta-regression analysis of referral rate

\begin{tabular}{|c|c|c|c|}
\hline Variable & $\mathrm{N}$ & Regression coefficient $(95 \% \mathrm{Cl})$ & Statistical significance $(p)$ \\
\hline Study country ${ }^{a}$ & & \multirow{3}{*}{$\begin{array}{l}\text { Reference: Low- and Middle- income country } \\
-0.16(-0.50,0.19)\end{array}$} & \multirow[t]{3}{*}{0.38} \\
\hline Low- and Middle- income country & 2 & & \\
\hline High-income country or region & 42 & & \\
\hline Time points of screening & & \multirow{4}{*}{$\begin{array}{l}\text { Reference: Prenatal } \\
0.04(-0.08,0.16)\end{array}$} & \multirow[t]{4}{*}{0.53} \\
\hline Prenatal & 8 & & \\
\hline Postnatal & 28 & & \\
\hline Perinatal & 8 & & \\
\hline Referral methods & & \multirow{3}{*}{$\begin{array}{l}\text { Reference: Referral to mental health service } \\
0.27(0.12,0.42)\end{array}$} & \multirow[t]{3}{*}{$<0.01^{\star}$} \\
\hline Referral to mental health service & 27 & & \\
\hline On-site assessment or treatment & 17 & & \\
\hline Number of referral interventions & & \multirow{6}{*}{$\begin{array}{l}\text { Reference: No intervention } \\
<0.01(-0.06,0.08)\end{array}$} & \multirow[t]{6}{*}{0.78} \\
\hline 0 & 4 & & \\
\hline 1 & 17 & & \\
\hline 2 & 14 & & \\
\hline 3 & 5 & & \\
\hline 4 & 4 & & \\
\hline
\end{tabular}

${ }^{a}$ Income based on World Bank classifications.

${ }^{*} p<0.05$.

Table 3. The nature of referral interventions among included studies

\begin{tabular}{|c|c|}
\hline Interventions & Definition \\
\hline Education & $\begin{array}{l}\text { Education on the definition, symptom and hazard of perinatal depression, prenatal depression or postnatal } \\
\text { depression for women with positive screening results, mostly in the form of brochures (Miller et al., 2004; Flynn } \\
\text { et al., 2006; Chen et al., 2011; Wisner et al., 2013; Bina, 2014; Boyd et al., 2015; BenDavid et al., 2016; Trost et al., } \\
\text { 2016; Bauer et al., 2017; Jarvis et al., 2018). }\end{array}$ \\
\hline On-site assessment or treatment & $\begin{array}{l}\text { Mental health assessment or treatment at current clinic, mainly provided by perinatal care provider (eg, obstetrician or } \\
\text { midwife), on-site social worker or researcher (Fergerson et al., 2002; Chaudron et al., 2004; Carter et al., 2005; Flynn } \\
\text { et al., 2006; Ingadottir and Thome, 2006; Harvey and Pun, 2007; Horowitz et al., 2009; Miller et al., 2009; Chen et al., } \\
\text { 2011; Leung et al., 2011; Honikman et al., 2012; Miller et al., 2012; Segre et al., 2012; Stock et al., 2013; Wisner et al., } \\
\text { 2013; Mestad et al., 2016; Venkatesh et al., 2016). }\end{array}$ \\
\hline Provision of resources & $\begin{array}{l}\text { Providing mental health resources to women with positive screening results, mostly in the forms of a handout of } \\
\text { PND resources (Miller et al., 2004; Reay et al., 2011; Stock et al., 2013; Emerson et al., 2014; BenDavid et al., 2016; } \\
\text { Trost et al., 2016; Bauer et al., 2017; Jarvis et al., 2018; Kallem et al., 2019). }\end{array}$ \\
\hline Health consultation or diagnosis & $\begin{array}{l}\text { Mental health consultation or diagnosis for women with positive screening results provided by a mental health } \\
\text { provider or social worker (Robinson and Young, 1982; Tam et al., 2002; Gordon et al., 2006; Orhon et al., 2007; } \\
\text { Horowitz et al., 2009; Burton et al., 2011; Segre et al., 2012; Stock et al., 2013; Emerson et al., 2014; Trost et al., 2016; } \\
\text { Jarvis et al., 2018; Lydsdottir et al., 2019). }\end{array}$ \\
\hline $\begin{array}{l}\text { Supportive treatment or referral to } \\
\text { support group }\end{array}$ & $\begin{array}{l}\text { Offering women with positive screening results in individual treatment (eg, psychotherapy or crisis intervention) or } \\
\text { inviting women with positive screening results to participate in PND support group (Ingadottir and Thome, 2006; } \\
\text { Chen et al., 2011; Milgrom et al., 2011; Segre et al., 2012; Jarvis et al., 2018). }\end{array}$ \\
\hline Assistance in referral & $\begin{array}{l}\text { Assistance by health care provider for mental health specialist appointment or further assessment or treatment (Miller } \\
\text { et al., 2004; Flynn et al., 2006; Gordon et al., 2006; Horowitz and Cousins, 2006; Horowitz et al., 2009; Reay et al., 2011; } \\
\text { Rowan et al., 2012; Nelson et al., 2013; Boyd et al., 2015; BenDavid et al., 2016; Venkatesh et al., 2016; Kallem et al., 2019). }\end{array}$ \\
\hline Health care provider training & $\begin{array}{l}\text { Training of health care provider in the knowledge and practice on screening, diagnosis, referral and management } \\
\text { (Gordon et al., 2006; Miller et al., 2009; Milgrom et al., 2011; Honikman et al., 2012; Miller et al., 2012; Segre et al., } \\
\text { 2012; Yawn et al., 2012; Wisner et al., 2013; BenDavid et al., 2016; Trost et al., 2016). }\end{array}$ \\
\hline
\end{tabular}

\section{Comparison with other studies and guidelines}

Compared to Byatt et al. (2015) and Long et al. (2019), we focused on the uptake rate and the effectiveness of interventions. We showed that nearly $60 \%$ of women with positive screening results do not take up the offers for referrals to psychological/psychiatric services after screening. This uptake rate is considerably lower than those in other screening programs, such as cancer screening (Yabroff et al., 2003; Callen et al., 2012; Dalton, 2018). Such a low 
Table 4. Characteristics of the experimental study included

\begin{tabular}{|c|c|c|c|c|c|c|c|}
\hline $\begin{array}{l}\text { Author } \\
\text { (year) }\end{array}$ & $\begin{array}{l}\text { Study type, } \\
\text { country and } \\
\text { year of } \\
\text { study }\end{array}$ & $\begin{array}{l}\text { Screening } \\
\text { tool and time } \\
\text { points }\end{array}$ & $\begin{array}{l}\text { Referral } \\
\text { methods }\end{array}$ & Grouping & Referral interventions & $\begin{array}{l}\text { Sample size, } \\
\text { uptake of } \\
\text { referral and } \\
\text { uptake rate }\end{array}$ & $\chi^{2}, p$ \\
\hline \multirow[t]{2}{*}{$\begin{array}{l}\text { Yawn } \\
\text { et al. } \\
(2012)\end{array}$} & \multirow[t]{2}{*}{$\begin{array}{l}\text { Cluster } \\
\text { RCTs; USA; } \\
\text { 2006-2010 }\end{array}$} & \multirow{2}{*}{$\begin{array}{l}\text { EPDS, } \\
\text { PHQ-9; 5-12 } \\
\text { weeks } \\
\text { postnatal }\end{array}$} & $\begin{array}{l}\text { Referral to } \\
\text { mental health } \\
\text { service }\end{array}$ & $\begin{array}{l}\text { Control } \\
\text { group }\end{array}$ & $\begin{array}{l}\text { Short health care } \\
\text { provider training }\end{array}$ & $233 ; 78 ; 33 \%$ & \multirow[t]{2}{*}{$\begin{array}{l}38.77 \\
<0.01^{\star}\end{array}$} \\
\hline & & & $\begin{array}{l}\text { On-site } \\
\text { assessment or } \\
\text { treatment }\end{array}$ & $\begin{array}{l}\text { Intervention } \\
\text { group }\end{array}$ & $\begin{array}{l}\text { On-site assessment, } \\
\text { health care provider } \\
\text { training, provision of } \\
\text { tools to facilitate the } \\
\text { management of PND }\end{array}$ & $322 ; 194 ; 60 \%$ & \\
\hline
\end{tabular}

${ }^{\star} p<0.05$.

uptake rate will reduce the utility of screening programs. The low uptake raises the importance of finding the reasons for it and developing the strategy for improvement. We note that government or professional bodies in many high-income countries have recommended screening for PND (Appendix E). For seven of them, only five included any comments on the challenge presented by a low uptake rate or suggestions on how this can be improved. Our findings suggest that it is an important oversight that needs to be addressed.

Among the different methods of referral, on-site assessment or treatment appeared to be more effective. Previous studies have shown that women who received referrals to the same site as their prenatal or postnatal care were more likely than those referred offsite (Smith et al., 2009; Flynn et al., 2010; Price et al., 2017). Access to further assessments and treatment on-site is more convenient and would reduce the degree of stigma and therefore more acceptable to women. To achieve on-site referral, mental health services are needed in the perinatal health care settings. However, collocated mental health professionals are likely to be absent in almost all resource-poor areas (Patel and Prince, 2010). Equipping perinatal health providers with the capacity of providing basic mental health services may be a practical option. It is important to note that among the 40 eligible observational or quasi-experimental studies in our review, all but two were conducted in high-income countries or regions. As epidemiological evidence indicates PND is more common in low-and middleincome countries (Akhtar and Landeen, 2007; Shidhaye and Giri, 2014), and that many of these countries have huge populations (Patel and Prince, 2010), this inequity needs to be addressed urgently. An important global health priority would, therefore, be to conduct locally relevant research in low-and middle-income countries, especially the evaluation of the feasibility and costeffectiveness of approaches to provide on-site assessment and treatment (for example, the use of mobile health technology or the training of perinatal care providers).

We found that the quality of the evidence on the effectiveness of the interventions to increase uptake was weak, as there was only one RCT that showed 'high risk' in performance bias. Furthermore, we note that interventions examined in previous studies tended to lack considerations for support from the woman's family, which was found to influence women's response to referral (Dennis, 2005; Ahmed et al., 2008). Another important consideration in improving the design of interventions is to collect qualitative information on the barriers to mental health services. In this review, among the two more frequent reasons mentioned were 'lack of time' and 'perception that mood had improved'. The former may be addressed by improving the accessibility of health care services (including time, costs and transportation) (Lara-Cinisomo et al., 2014; Nagle and Farrelly, 2018; Jones, 2019). As the changes in circumstances for women and their families in the perinatal period can often be overwhelming, convenience when designing interventions would be important. The latter may reflect beliefs and cultural attitudes that may be modified by interventions aimed at improving knowledge about depression (Sword et al., 2008; Canty et al., 2019).

\section{Strengths and limitations}

Strengths of this review included: first, our research question focused on the uptake of referral by women with positive PND screening results which has not been reported in previous reviews; second, we examined the effect of interventions and reasons of non-uptake to inform screening programs as well the design of further research to increase uptake of referral. Our review has several limitations. First, in the protocol registered with PROSPERO, the subject of the review is on referral in general. For reasons explained in detail in the Introduction, this review focuses on uptake rather than all three steps in the referral and treatment process. Second, the heterogeneity for the pooled uptake rate was high across the eligible studies. Only the referral method was identified as a moderator of the observed heterogeneity, suggesting that future studies should further explore the factors that contribute to the high heterogeneity. Third, the quality of the included studies was an important limitation in estimating the uptake of referral and assessing the effectiveness of interventions to increase uptake, with only one RCT in 41 eligible studies. Fourth, we searched for publications in English and Chinese only and those that would have missed articles in other languages. Finally, we only included information from published studies when assessing uptake rates. As we do not have information from the ongoing services worldwide, we cannot exclude the possibility that the uptake rates from these services may be higher than those reported in published studies.

\section{Implications}

We find that almost three-fifths of women with positive perinatal depression screening results do not take up the offers for referrals to mental health service. In countries where screening is recommended, the reasons behind this low uptake should be assessed. Though efforts to address the challenge will be hampered by the weak overall quality of evidence on interventions to increase 
uptake, there is some suggestion that referral to on-site assessment and treatment may be helpful. Finally, as little is known in low-and middle-income countries where most affected women live, this issue should be addressed as an important global health research priority.

Data. All the data supporting the findings of this meta-analysis have been provided in Tables and Figures.

Acknowledgements. We thank Lu Liu, Qiao Li, Jie Wang, Ping Ye and Xiaodi Ma, all from Central South University, for literature searches, screened articles for inclusion and extracted data.

Financial support. This work was funded by The National Nature Science Foundation of China (grant No 81773446, 81402690).

Conflict of interest. None.

Ethical standards. This research did not involve human and/or animal experimentation.

\section{References}

ACOG (2018) ACOG committee opinion No. 757: screening for perinatal depression. Obstetrics \& Gynecology 132, e208-e212.

Ahmed A, Stewart DE, Teng L, Wahoush $\mathbf{O}$ and Gagnon AJ (2008) Experiences of immigrant new mothers with symptoms of depression. Archives of Women's Mental Health 11, 295-303.

Akhtar D and Landeen J (2007) Relation between depression and sociodemographic factors. International Journal of Mental Health Systems 1, 4.

Bauer NS, Ofner S, Pottenger A, Carroll AE and Downs SM (2017) Follow-up of mothers with suspected postpartum depression from pediatrics clinics. Frontiers in Pediatrics 5, 212.

Beardslee WR, Versage EM and Gladstone TR (1998) Children of affectively ill parents: a review of the past 10 years. Journal of the American Academy of Child and Adolescent Psychiatry 37, 1134-1141.

Beck AT, Ward CH, Mendelson M, Mock J and Erbaugh J (1961) An inventory for measuring depression. Archives of General Psychiatry 4, 561-571.

BenDavid DN, Hunker DF and Spadaro KC (2016) Uncovering the golden veil: applying the evidence for telephone screening to detect early postpartum depression. Journal of Perinatal Education 25, 37-45.

Bina R (2014) Seeking help for postpartum depression in the Israeli Jewish orthodox community: factors associated with use of professional and informal help. Women \& Health 54, 455-473.

Boyd RC, Mogul M and O'Hara MW (2015) A pilot investigation to enhance behavioral health referral for perinatal, Low-income women with mood disorders. Progress in Community Health Partnerships 9, 583-589.

Buist AE, Austin MP, Hayes BA, Speelman C, Bilszta JL, Gemmill AW, Brooks J, Ellwood D and Milgrom J (2008) Postnatal mental health of women giving birth in Australia 2002-2004: findings from the beyond blue National Postnatal Depression Program. Australian and New Zealand Journal of Psychiatry 42, 66-73.

Burton A, Patel S, Kaminsky L, Rosario GD, Young R, Fitzsimmons A and Canterino JC (2011) Depression in pregnancy: time of screening and access to psychiatric care. Journal of Maternal-Fetal \& Neonatal Medicine 24, 1321-1324.

Byatt N, Levin LL, Ziedonis D, Moore-Simas TA and Allison J (2015) Enhancing participation in depression care in outpatient perinatal care settings: a systematic review. Obstetrics \& Gynecology 126, 1048-1058.

Callen JL, Westbrook JI, Georgiou A and Li J (2012) Failure to follow-up test results for ambulatory patients: a systematic review. Journal of General Internal Medicine 27, 1334-1348.

Canty HR, Sauter A, Zuckerman K, Cobian M and Grigsby T (2019) Mothers' perspectives on follow-up for postpartum depression screening in primary care. Journal of Developmental and Behavioral Pediatrics 40, 139-143.

Carter FA, Carter JD, Luty SE, Wilson DA, Frampton CM and Joyce PR (2005) Screening and treatment for depression during pregnancy: a cautionary note. Australian and New Zealand Journal of Psychiatry 39, 255-261.

Chaudron LH, Szilagyi PG, Kitzman HJ, Wadkins HI and Conwell Y (2004) Detection of postpartum depressive symptoms by screening at well-child visits. Pediatrics 113, 551-558.

Chen H, Wang J, Ch'ng YC, Mingoo R, Lee T and Ong J (2011) Identifying mothers with postpartum depression early: integrating perinatal mental health care into the obstetric setting. ISRN Obstetrics and Gynecology 2011, 309189.

Cox JL, Holden JM and Sagovsky R (1987) Detection of postnatal depression. Development of the 10-item Edinburgh Postnatal Depression Scale. British Journal of Psychiatry 150, 782-786.

Dalton ARH (2018) Incomplete diagnostic follow-up after a positive colorectal cancer screening test: a systematic review. Journal of Public Health (Oxf) 40, e46-e58.

Dennis CL (2005) Psychosocial and psychological interventions for prevention of postnatal depression: systematic review. The British Medical Journal 331, 15.

Earls MF, Committee on Psychosocial Aspects of Child and Family Health American Academy of Pediatrics (2010) Incorporating recognition and management of perinatal and postpartum depression into pediatric practice. Pediatrics 126, 1032-1039.

Emerson BL, Bradley ER, Riera A, Mayes L and Bechtel K (2014) Postpartum depression screening in the pediatric emergency department. Pediatric Emergency Care 30, 788-792.

Fergerson SS, Jamieson DJ and Lindsay M (2002) Diagnosing postpartum depression: can we do better? American Journal of Obstetrics \& Gynecology 186, 899-902.

Flynn HA, O'Mahen HA, Massey L and Marcus S (2006) The impact of a brief obstetrics clinic-based intervention on treatment use for perinatal depression. Journal of Women's Health (Larchmt) 15, 1195-1204.

Flynn HA, Henshaw E, O'Mahen H and Forman J (2010) Patient perspectives on improving the depression referral processes in obstetrics settings: a qualitative study. General Hospital Psychiatry 32, 9-16.

Freeman MF and Turkey JW (1950) Transformations related to the angular and the square root. The Annals of Mathematical Statistics 21, 607-611.

Gajaria A and Ravindran AV (2018) Interventions for perinatal depression in low and middle-income countries: a systematic review. Asian Journal of Psychiatry 37, 112-120.

Gavin NI, Gaynes BN, Lohr KN, Meltzer-Brody S, Gartlehner G and Swinson T (2005) Perinatal depression: a systematic review of prevalence and incidence. Obstetrics \& Gynecology 106, 1071-1083.

Goodman SH, Rouse MH, Connell AM, Broth MR, Hall CM and Heyward D (2011) Maternal depression and child psychopathology: a meta-analytic review. Clinical Child and Family Psychology Review 14, 1-27.

Gordon TE, Cardone IA, Kim JJ, Gordon SM and Silver RK (2006) Universal perinatal depression screening in an Academic Medical Center. Obstetrics \& Gynecology 107, 342-347.

Harvey ST and Pun PK (2007) Analysis of positive Edinburgh depression scale referrals to a consultation liaison psychiatry service in a two-year period. International Journal of Mental Health Nursing 16, 161-167.

Hewitt CE and Gilbody SM (2009) Is it clinically and cost effective to screen for postnatal depression: a systematic review of controlled clinical trials and economic evidence. British Journal of Obstetrics and Gynecology 116, 1019-1027.

Higgins JP, Altman DG, Gotzsche PC, Juni P, Moher D, Oxman AD, Savovic J, Schulz KF, Weeks L and Sterne JA, Cochrane Bias Methods Group; Cochrane Statistical Methods Group (2011) The Cochrane Collaboration's tool for assessing risk of bias in randomised trials. The British Medical Journal 343, d5928.

Honikman S, van-Heyningen T, Field S, Baron E and Tomlinson M (2012) Stepped care for maternal mental health: a case study of the perinatal mental health project in South Africa. PLoS Medicine 9, e1001222.

Horowitz JA and Cousins A (2006) Postpartum depression treatment rates for at-risk women. Nursing Research 55, S23-S27. 
Horowitz JA, Murphy CA, Gregory KE and Wojcik J (2009) Best practices: community-based postpartum depression screening: results from the CARE study. Psychiatric Services 60, 1432-1434.

Ingadottir E and Thome M (2006) Evaluation of a web-based course for community nurses on postpartum emotional distress. Scandinavian Journal of Caring Sciences 20, 86-92.

Jarvis LR, Breslin KA, Badolato GM, Chamberlain JM and Goyal MK (2018) Postpartum depression screening and referral in a pediatric emergency department. Pediatric Emergency Care doi: 10.1097/ PEC.0000000000001499.

Jones A (2019) Help seeking in the perinatal period: a review of barriers and facilitators. Social Work in Public Health 34, 596-605.

Kallem S, Matone M, Boyd RC and Guevara JP (2019) Mothers' mental health care Use after screening for postpartum depression at well-child visits. Academic Pediatrics 19, 652-658.

Kim JJ, La Porte LM, Corcoran M, Magasi S, Batza J and Silver RK (2010) Barriers to mental health treatment among obstetric patients at risk for depression. American Journal of Obstetrics \& Gynecology 202, 312 e311-315.

Lara-Cinisomo S, Wisner KL, Burns RM and Chaves-Gnecco D (2014) Perinatal depression treatment preferences among Latina mothers. Qualitative Health Research 24, 232-241.

Leung SS, Leung C, Lam TH, Hung SF, Chan R, Yeung T, Miao M, Cheng S, Leung SH, Lau A and Lee DT (2011) Outcome of a postnatal depression screening programme using the Edinburgh postnatal depression scale: a randomized controlled trial. Journal of Public Health (Oxf 33, 292-301.

Loney PL, Chambers LW, Bennett KJ, Roberts JG and Stratford PW (1998) Critical appraisal of the health research literature: prevalence or incidence of a health problem. Chronic Disease in Canada 19, 170-176.

Long MM, Cramer RJ, Jenkins J, Bennington L and Paulson JF (2019) A systematic review of interventions for healthcare professionals to improve screening and referral for perinatal mood and anxiety disorders. Archives of Women's Mental Health 22, 25-36.

Lovibond PF and Lovibond SH (1995) The structure of negative emotional states: comparison of the Depression Anxiety Stress Scales (DASS) with the Beck Depression and Anxiety Inventories. Behaviour Research and Therary 33, 335-343.

Luo ML, Tan HZ, Zhou Q, Wang SY, Chang C, Guo YW and Shen L (2013) Realizing the meta-analysis of single rate in $\mathrm{R}$ software. The Journal of Evidence-Based Medicine 13, 181-184.

Lydsdottir LB, Howard LM, Olafsdottir H, Thome M, Tyrfingsson P and Sigurdsson JF (2019) The psychometric properties of the Icelandic version of the Edinburgh Postnatal Depression Scale (EPDS) when used prenatal. Midwifery 69, 45-51.

Mestad R, Lane SD, Hall M, Smith CJ, Carter DB, Rubinstein RA, Keefe RH and Jones-Moore C (2016) Prenatal depression: screening and referral for women who are low income during antenatal care. Social Work in Public Health 31, 557-564.

Milgrom J and Gemmill AW (2014) Screening for perinatal depression. Best Practice and Research. Clinical Obstetrics and Gynaecology 28, 13-23.

Milgrom J, Holt CJ, Gemmill AW, Ericksen J, Leigh B, Buist A and Schembri C (2011) Treating postnatal depressive symptoms in primary care: a randomised controlled trial of GP management, with and without adjunctive counselling. BMC Psychiatry 11, 95.

Miller L, Boyd B and Chernov A (2004) Improving the identification and treatment of postpartum depression in a managed care organization. Journal of Clinical Outcomes Management 11, 157-161.

Miller L, Shade M and Vasireddy V (2009) Beyond screening: assessment of perinatal depression in a perinatal care setting. Archives of Women's Mental Health 12, 329-334.

Miller LJ, McGlynn A, Suberlak K, Rubin LH, Miller M and Pirec V (2012) Now what? Effects of on-site assessment on treatment entry after perinatal depression screening. Journal of Women's Health (Larchmt) 21, 1046-1052.

Muzik M and Borovska S (2010) Perinatal depression: implications for child mental health. Mental Health in Family Medicine 7, 239-247.
Nagle U and Farrelly M (2018) Women's views and experiences of having their mental health needs considered in the perinatal period. Midwifery 66, 79-87.

Nelson DB, Freeman MP, Johnson NL, McIntire DD and Leveno KJ (2013) A prospective study of postpartum depression in 17648 parturients. Journal of Maternal-Fetal \& Neonatal Medicine 26, 1155-1161.

O'Connor E, Rossom RC, Henninger M, Groom HC, Burda BU, Henderson JT, Bigler KD and Whitlock EP (2016). Screening for Depression in Adults: An Updated Systematic Evidence Review for the U.S. Preventive Services Task Force. Rockville, MD.

Orhon FS, Soykan A and Ulukol B (2007) Patient compliance to psychiatric interventions and course of postpartum mood disorders. International Journal of Psychiatry in Medicine 37, 445-457.

Patel V and Prince M (2010) Global mental health: a new global health field comes of age. The Journal of American Medical Association 303, 1976-1977.

Price SK, Corder-Mabe J and Austin K (2012) Perinatal depression screening and intervention: enhancing health provider involvement. Journal of Womens Health (Larchmt) 21, 447-455.

Price SK, Coles DC and Wingold T (2017) Integrating behavioral health risk assessment into centralized intake for maternal and child health services. Health and Social Work 42, 231-240.

Reay R, Matthey S, Ellwood D and Scott M (2011) Long-term outcomes of participants in a perinatal depression early detection program. Journal of Affective Disorder 129, 94-103.

Rhonda S, Stephanie B and Judith LJA (1994) Missing voices: what women say and do about depression after childbirth. Journal of Reproductive and Infant Psychology 12, 89-103.

Robinson S and Young J (1982) Screening for depression and anxiety in the post-natal period: acceptance or rejection of a subsequent treatment offer. Australian and New Zealand Journal of Psychiatry 16, 47-51.

Rowan P, Greisinger A, Brehm B, Smith F and McReynolds E (2012) Outcomes from implementing systematic antepartum depression screening in obstetrics. Archives of Women's Mental Health 15, 115-120.

Savovic J, Turner RM, Mawdsley D, Jones HE, Beynon R, Higgins JPT and Sterne JAC (2018) Association between risk-of-bias assessments and results of randomized trials in Cochrane reviews: the ROBES meta-epidemiologic study. American Journal of Epidemiology 187, 1113-1122.

Segre LS, O'Hara MW, Brock RL and Taylor D (2012) Depression screening of perinatal women by the Des Moines Healthy Start Project: program description and evaluation. Psychiatric Services 63, 250-255.

Shidhaye P and Giri P (2014) Maternal depression: a hidden burden in developing countries. Annals of Medical and Health Sciences Research 4, 463465.

Smith MV, Shao L, Howell H, Wang H, Poschman K and Yonkers KA (2009) Success of mental health referral among pregnant and postpartum women with psychiatric distress. General Hospital Psychiatry 31, 155-162.

Snaith RP, Bridge GW and Hamilton M (1976) The Leeds scales for the selfassessment of anxiety and depression. British Journal of Psychiatry 128 156-165.

Stock A, Chin L, Babl FE, Bevan CA, Donath S and Jordan B (2013) Postnatal depression in mothers bringing infants to the emergency department. Archives of Disease in Childhood 98, 36-40.

Sword W, Busser D, Ganann R, McMillan T and Swinton M (2008) Women's care-seeking experiences after referral for postpartum depression. Qualitative Health Research 18, 1161-1173.

Tam LW, Newton RP, Dern M and Parry BL (2002) Screening women for postpartum depression at well baby visits: resistance encountered and recommendations. Archives of Women's Mental Health 5, 79-82.

The World Bank Group (2016). Country and Lending Groups. https:// datahelpdesk.worldbank.org/knowledgebase/articles/906519.

Thombs BD, Arthurs E, Coronado-Montoya S, Roseman M, Delisle VC, Leavens A, Levis B, Azoulay L, Smith C, Ciofani L, Coyne JC, Freeley N, Gilbody S, Schinazi J, Stewart DE and Zelkowitz P (2014) Depression screening and patient outcomes in pregnancy or postpartum: a systematic review. Journal of Psychosomatic Research 76, 433-446.

Trost MJ, Molas-Torreblanca K, Man C, Casillas E, Sapir H and Schrager SM (2016) Screening for maternal postpartum depression during infant hospitalizations. Journal of Hospital Medicine 11, 840-846. 
Urato AC (2017) Consensus bundle on maternal mental health: perinatal depression and anxiety. Obstetrics \& Gynecology 130, 467-468.

Venkatesh KK, Nadel H, Blewett D, Freeman MP, Kaimal AJ and Riley LE (2016) Implementation of universal screening for depression during pregnancy: feasibility and impact on obstetric care. American Journal of Obstetrics \& Gynecology 215, e511-e518.

Wisner KL, Sit DK, McShea MC, Rizzo DM, Zoretich RA, Hughes CL, Eng HF, Luther JF, Wisniewski SR, Costantino ML, Confer AL, Moses-Kolko EL, Famy CS and Hanusa BH (2013) Onset timing, thoughts of self-harm, and diagnoses in postpartum women with screen-positive depression findings. JAMA psychiatry 70, 490-498.
Wittkampf KA, Naeije L, Schene AH, Huyser J and van Weert HC (2007) Diagnostic accuracy of the mood module of the Patient Health Questionnaire: a systematic review. General Hospital Psychiatry 29, 388-395.

Yabroff KR, Washington KS, Leader A, Neilson E and Mandelblatt J (2003) Is the promise of cancer-screening programs being compromised? Quality of follow-up care after abnormal screening results. Medical Care Research and Review 60, 294-331.

Yawn BP, Dietrich AJ, Wollan P, Bertram S, Graham D, Huff J, Kurland M, Madison S and Pace WD (2012) TRIPPD: a practice-based network effectiveness study of postpartum depression screening and management. Annals of Family Medicine 10, 320-329.

\section{Appendix A: Search Strategy}

Search Strategy (PubMed, -2019/01)

\begin{tabular}{lll}
\hline Step & \multicolumn{1}{c}{ Category } & \multicolumn{1}{c}{ Terms } \\
\hline 1 & Terms for 'perinatal' & $\begin{array}{l}\text { (perinatal[all] OR pregnant[all] OR pregnancy[all] OR prenatal[all] OR antenatal } \\
\text { [all] OR postnatal[all] OR postpartum[all]) }\end{array}$ \\
\hline 2 & Terms for 'depression' & (depression[all] OR depressive symptoms[all]) \\
\hline 3 & Terms for 'screening' & screening[all] \\
\hline 4 & Terms for 'referral' & (referral[all] OR referrals[all] OR refer[all] OR transfer[all] OR uptake[all]) \\
\hline 5 & $\begin{array}{l}\text { Applies limits to combined 'perinatal' and 'depression' and } \\
\text { 'screening' and 'referral' }\end{array}$ & \#1 AND \#2 AND \#3 AND \#4 \\
\hline
\end{tabular}

Search Strategy (Cochrane Library, -2019/01)

\begin{tabular}{lll}
\hline Step & \multicolumn{1}{c}{ Category } & \multicolumn{1}{c}{ Terms } \\
\hline 1 & Terms for 'perinatal' & $\begin{array}{l}\text { (perinatal[ts] OR pregnant[ts] OR pregnancy[ts] OR prenatal[ts] OR antenatal[ts] } \\
\text { OR postnatal[ts] OR postpartum[ts]) }\end{array}$ \\
\hline 2 & Terms for 'depression' & (depression[ts] OR depressive symptoms[ts]) \\
\hline 3 & Terms for 'screening' & screening[ts] \\
\hline 4 & Terms for 'referral' & (referral[ts] OR referrals[ts] OR refer[ts] OR transfer[ts] OR uptake[ts]) \\
\hline 5 & $\begin{array}{l}\text { Applies limits to combined 'perinatal' and 'depression' and } \\
\text { 'screening' and 'referral' }\end{array}$ & \#1 AND \#2 AND \#3 AND \#4 \\
\hline
\end{tabular}

Search Strategy (Web of Science, -2019/01)

\begin{tabular}{lll}
\hline Step & \multicolumn{1}{c}{ Category } & \multicolumn{1}{c}{ Terms } \\
\hline 1 & Terms for 'perinatal' & $\begin{array}{l}\text { (perinatal[ts] OR pregnant[ts] OR pregnancy[ts] OR prenatal[ts] OR antenatal[ts] } \\
\text { OR postnatal[ts] OR postpartum[ts]) }\end{array}$ \\
\hline 2 & Terms for 'depression' & (depression[ts] OR depressive symptoms[ts]) \\
\hline 3 & Terms for 'screening' & screening[ts] \\
\hline 4 & Terms for 'referral' & (referral[ts] OR referrals[ts] OR refer[ts] OR transfer[ts] OR uptake[ts]) \\
\hline 5 & $\begin{array}{l}\text { Applies limits to combined 'perinatal' and 'depression' and } \\
\text { 'screening' and 'referral' }\end{array}$ & \#1AND \#2 AND \#3 AND \#4 \\
\hline
\end{tabular}

Search Strategy (Embase, -2019/01)

\begin{tabular}{lll}
\hline Step & \multicolumn{1}{c}{ Category } & \multicolumn{1}{c}{ Terms } \\
\hline 1 & Terms for 'perinatal' & $\begin{array}{l}\text { (perinatal[all] OR pregnant[all] OR pregnancy[all] OR prenatal[all] OR antenatal } \\
\text { [all] OR postnatal[all] OR postpartum[all]) }\end{array}$ \\
\hline 2 & Terms for 'depression' & (depression[all] OR depressive symptoms[all]) \\
\hline 3 & Terms for 'screening' & screening[all] \\
\hline 4 & Terms for 'referral' & (referral[all] OR referrals[all] OR refer[all] OR transfer[all] OR uptake[all]) \\
\hline 5 & $\begin{array}{l}\text { Applies limits to combined 'perinatal' and 'depression' and } \\
\text { 'screening' and 'referral' }\end{array}$ & \#1 AND \#2 AND \#3 AND \#4 \\
\hline
\end{tabular}


Search Strategy (Ovid, -2019/01)

\begin{tabular}{lll}
\hline Step & \multicolumn{1}{c}{ Category } & \multicolumn{1}{c}{ Terms } \\
\hline 1 & Terms for 'perinatal' & $\begin{array}{l}\text { (perinatal[mp] OR pregnant[mp] OR pregnancy[mp] OR prenatal[mp] OR antenatal } \\
\text { [mp] OR postnatal[mp] OR postpartum[mp]) }\end{array}$ \\
\hline 2 & Terms for 'depression' & (depression[mp] OR depressive symptoms[mp]) \\
\hline 3 & Terms for 'screening' & screening[mp] \\
\hline 4 & Terms for 'referral' & (referral[mp] OR referrals[mp] OR refer[mp] OR transfer[mp] OR uptake[mp]) \\
\hline 5 & $\begin{array}{l}\text { Applies limits to combined 'perinatal' and 'depression' } \\
\text { and 'screening' and 'referral' }\end{array}$ & \#1 AND \#2 AND \#3 AND \#4 \\
\hline
\end{tabular}

Search Strategy (CNKI, -2019/01)

\begin{tabular}{|c|c|c|}
\hline Step & Category & Terms \\
\hline
\end{tabular}

Search Strategy (Wanfang, -2019/01)

\begin{tabular}{|c|c|c|}
\hline Step & Category & Terms \\
\hline 1 & Applies limits to combined ‘围产期’ and ‘抑郁” and ‘篮查” and “转诊” & 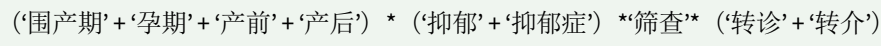 \\
\hline
\end{tabular}

Search Strategy (VIP, -2019/01)

\begin{tabular}{lll}
\hline Step & \multicolumn{1}{c}{ Category } & Terms \\
\hline 1 & $\begin{array}{l}\text { Applies limits to combined '围产期” and “抑有” and '笁 } \\
\text { 查' and “转诊” }\end{array}$ & $\begin{array}{l}U=\text { (围产期 OR 孕期 OR 产前 OR 产后) AND U = (抑郁 OR 抑有症) AND U = 笁查 AND } \\
U=\text { (转诊 OR 转介) }\end{array}$ \\
\hline
\end{tabular}

\section{Appendix B: Results of quality assessment of the included RCT}

\begin{tabular}{|c|c|c|c|c|c|c|c|}
\hline & \multicolumn{2}{|c|}{ Selection bias } & \multirow[b]{2}{*}{$\begin{array}{l}\text { Performance } \\
\text { bias }\end{array}$} & \multirow[b]{2}{*}{$\begin{array}{l}\text { Detection } \\
\text { bias }\end{array}$} & \multirow[b]{2}{*}{$\begin{array}{l}\text { Attrition } \\
\text { bias }\end{array}$} & \multirow[b]{2}{*}{$\begin{array}{l}\text { Reporting } \\
\text { bias }\end{array}$} & \multirow[b]{2}{*}{$\begin{array}{l}\text { Other } \\
\text { bias }\end{array}$} \\
\hline & $\begin{array}{l}\text { Random sequence } \\
\text { generation }\end{array}$ & $\begin{array}{c}\text { Allocation } \\
\text { concealment }\end{array}$ & & & & & \\
\hline $\begin{array}{l}\text { Yawn et al. } \\
\text { (2012) }\end{array}$ & Unclear risk & Unclear risk & High risk & Unclear risk & Unclear risk & Unclear risk & Low risk \\
\hline
\end{tabular}

\section{Appendix C: Patient outcomes reported by included studies}

\begin{tabular}{|c|c|c|c|}
\hline Author (Year) & $\begin{array}{l}\text { Outcome } \\
\text { domain }\end{array}$ & Outcome measure & Patient outcomes \\
\hline $\begin{array}{l}\text { Venkatesh et al. } \\
\text { (2016) }\end{array}$ & Depression & EPDS & $\begin{array}{l}20 \% \text { women who 'screened positive antepartum and linked to mental } \\
\text { health services' experienced a reduction of scores to below the cut-off of } \\
12 \text {. }\end{array}$ \\
\hline Trost et al. (2016) & Depression & EPDS & $\begin{array}{l}\text { 'Of } 21 \text { mothers initially EPDS1 who completed a follow-up call, } 10(48 \%) \\
\text { later screened negative.' }\end{array}$ \\
\hline \multirow[t]{2}{*}{ Boyd et al. (2015) } & Depression & $\mathrm{BDI}$ & $\begin{array}{l}\text { 'Depression scores decreased significantly from baseline to } \\
\text { postintervention follow-up }(p<0.01) \text { ' }\end{array}$ \\
\hline & Social support & $\begin{array}{l}\text { Multidimensional Scale of } \\
\text { Perceived Social Support }\end{array}$ & 'No change in social support over time.' \\
\hline Segre et al. (2012) & Depression & EPDS & $\begin{array}{l}\text { 'Treatment recipients experienced a decline in depressive symptoms } \\
\text { between their first elevated EPDS score and their last available EPDS } \\
\text { score }(16.12 \pm 3.62 \text { v. } 10.50 \pm 5.73 ; p<0.001) \text {.' }\end{array}$ \\
\hline
\end{tabular}


Appendix C: (Continued.)

\begin{tabular}{|c|c|c|c|}
\hline Author (Year) & $\begin{array}{l}\text { Outcome } \\
\text { domain }\end{array}$ & Outcome measure & Patient outcomes \\
\hline Yawn et al. (2012) & Depression & PHQ-9 & $\begin{array}{l}\text { 'Among the } 654 \text { women with elevated postpartum depression screening } \\
\text { scores, those in the intervention practices had lower depressive symptom } \\
\text { levels at } 6(p=0.07) \text { and } 12 \text { months' }(p=0.001) \text { postpartum' }\end{array}$ \\
\hline \multirow[t]{2}{*}{$\begin{array}{l}\text { Milgrom et al. } \\
\text { (2011) }\end{array}$} & Depression & $\mathrm{BDI}$ & $\begin{array}{l}\text { 'This constituted a significant drop between baseline and post-study } \\
\text { (mean reduction in BDI-II scores for all treatment groups combined = } 17.3 \\
\text { points, } 95 \% \mathrm{Cl} 14.2-20.5 \text { )' }\end{array}$ \\
\hline & $\begin{array}{l}\text { Anxiety and } \\
\text { stress }\end{array}$ & DASS & $\begin{array}{l}\text { There were 'significant }(p<0.05) \text { overall drop in anxiety over the course of } \\
\text { the study.' }\end{array}$ \\
\hline \multirow[t]{3}{*}{ Chen et al. (2011) } & Depression & EPDS & $\begin{array}{l}\text { 'Of these } 41 \text { under clinical intervention, } 78 \%(32) \text { experiencing a reduction } \\
\text { of scores to below the cut-off score of } 13 . \text {.' }\end{array}$ \\
\hline & $\begin{array}{l}\text { Function and } \\
\text { symptoms }\end{array}$ & $\begin{array}{l}\text { Global Assessment of } \\
\text { Functioning Scale }\end{array}$ & '76\% (31) had a reduction in GAF scores.' \\
\hline & Health status & EuroQol health index & ' $68 \%(28)$ had a reduction in EQ-5D utility scores.' \\
\hline $\begin{array}{l}\text { Leung et al. } \\
\text { (2011) }\end{array}$ & Depression & EPDS & $\begin{array}{l}\text { At } 18 \text { months, there were } 132(80 \%) \text { participants from the intervention } \\
\text { and control groups with EPDS scores }<10 \text {. }\end{array}$ \\
\hline $\begin{array}{l}\text { Orhon et al. } \\
(2007)\end{array}$ & Depression & EPDS & $\begin{array}{l}\text { 'In the overall sample, EPDS scores decreased on average by } 7.4 \pm 4.7 \\
\text { points.' }\end{array}$ \\
\hline $\begin{array}{l}\text { Ingadottir and } \\
\text { Thome (2006) }\end{array}$ & Depression & EPDS & $\begin{array}{l}\text { Fifteen weeks after birth, nine of } 12 \text { women at the experimental CHCs } \\
\text { scored }<12 \text { on the EPDS but } 1 \text { of } 10 \text { scored }<12 \text { at control CHCs. } \\
\text { Twenty-four weeks after birth, seven of eight women at experimental } \\
\text { CHCs scored }<12 \text { on the EPDS but five of eight women scored }<12 \text { at } \\
\text { control CHCs. }\end{array}$ \\
\hline
\end{tabular}

\section{Appendix D: Funnel plot of the meta-analysis of referral uptake rates}

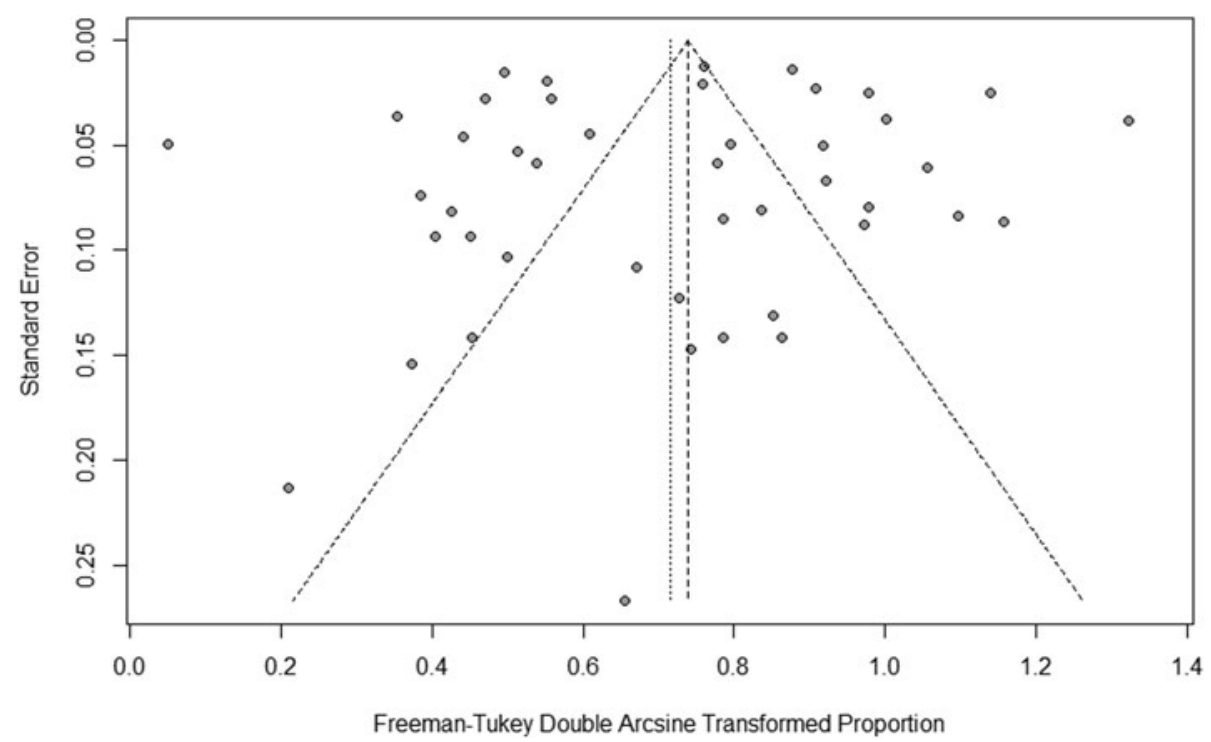


Appendix E: Recommendations from different organisations on perinatal depression screening

\begin{tabular}{|c|c|c|c|c|c|}
\hline Organisations & Condition & $\begin{array}{l}\text { Is it } \\
\text { recommended } \\
\text { to screening }\end{array}$ & Suggestions for screening & $\begin{array}{l}\text { Comments on the referral } \\
\text { and uptake of referral }\end{array}$ & References \\
\hline $\begin{array}{l}\text { National } \\
\text { Association of } \\
\text { Pediatric Nurse } \\
\text { Practitioners }\end{array}$ & $\begin{array}{l}\text { Postnatal } \\
\text { depression }\end{array}$ & $\begin{array}{l}\text { Screening } \\
\text { recommended }\end{array}$ & $\begin{array}{l}\text { All pediatric nurse } \\
\text { practitioners should be } \\
\text { skilful 'in screening } \\
\text { mothers for risk of } \\
\text { maternal depression' }\end{array}$ & None & $\begin{array}{l}\text { https://www. } \\
\text { sciencedirect.com/ } \\
\text { science/article/pii/ } \\
\text { S0891524510003159?via } \\
\text { \%3Dihub }\end{array}$ \\
\hline $\begin{array}{l}\text { US Preventive } \\
\text { Services Task Force }\end{array}$ & $\begin{array}{l}\text { Depression in } \\
\text { adults }\end{array}$ & $\begin{array}{l}\text { Screening } \\
\text { recommended }\end{array}$ & $\begin{array}{l}\text { 'Recommend screening } \\
\text { for depression in general } \\
\text { adults population, } \\
\text { including pregnant and } \\
\text { postpartum women. } \\
\text { Screening should be } \\
\text { implemented with } \\
\text { adequate systems in } \\
\text { place to ensure accurate } \\
\text { diagnosis, effective } \\
\text { treatment and follow-up.' }\end{array}$ & $\begin{array}{l}\text { The trial assessing the } \\
\text { effectiveness of screening } \\
\text { and treatment in older } \\
\text { adults 'had a number of } \\
\text { features that may affect } \\
\text { its reliability, including } \\
\text { external referrals for } \\
\text { depression treatment, } \\
\text { vert low uptake of } \\
\text { treatment (19\%), } \\
\text { suggesting that the }\end{array}$ & $\begin{array}{l}\text { 10.1001/ } \\
\text { jama.2015.18392 }\end{array}$ \\
\hline
\end{tabular}


Appendix E: (Continued.)

\begin{tabular}{|c|c|c|c|c|c|}
\hline Organisations & Condition & $\begin{array}{l}\text { Is it } \\
\text { recommended } \\
\text { to screening }\end{array}$ & Suggestions for screening & $\begin{array}{c}\text { Comments on the referral } \\
\text { and uptake of referral }\end{array}$ & References \\
\hline & & & $\begin{array}{l}\text { 'Adequate systems in } \\
\text { place' refers to having } \\
\text { systems and clinical staff } \\
\text { to ensure that patients } \\
\text { are } \\
\text { screened and, if they } \\
\text { screen positive, are } \\
\text { appropriately diagnosed } \\
\text { and treated with } \\
\text { evidence-based care or } \\
\text { referred to a setting that } \\
\text { can provide the } \\
\text { necessary care.' }\end{array}$ & $\begin{array}{l}\text { control and intervention } \\
\text { group may have been } \\
\text { different at baseline.' }\end{array}$ & \\
\hline $\begin{array}{l}\text { Mental Health } \\
\text { America }\end{array}$ & $\begin{array}{l}\text { Perinatal } \\
\text { depression }\end{array}$ & $\begin{array}{l}\text { Screening } \\
\text { recommended }\end{array}$ & $\begin{array}{l}\text { 'Screening for perinatal } \\
\text { mood and anxiety } \\
\text { disorders and follow up } \\
\text { care should be a required } \\
\text { element as part of health } \\
\text { home and general } \\
\text { medical and mental } \\
\text { health integration in all } \\
\text { health plans.' }\end{array}$ & $\begin{array}{l}\text { 'Mental health } \\
\text { professionals should be } \\
\text { co-located within the } \\
\text { settings where screening } \\
\text { is performed to provide } \\
\text { immediate evaluation, } \\
\text { diagnosis, and treatment } \\
\text { of mothers with positive } \\
\text { screening results. This } \\
\text { approach will reduce } \\
\text { barriers to care, improve } \\
\text { compliance, and provide } \\
\text { the best outcomes for } \\
\text { mothers and infants.' }\end{array}$ & $\begin{array}{l}\text { https://www. } \\
\text { mhanational.org/issues/ } \\
\text { position-statement-49- } \\
\text { perinatal-mental-health }\end{array}$ \\
\hline $\begin{array}{l}\text { The American } \\
\text { College of } \\
\text { Obstetricians and } \\
\text { Gynecologists }\end{array}$ & $\begin{array}{l}\text { Perinatal } \\
\text { depression }\end{array}$ & $\begin{array}{l}\text { Screening } \\
\text { recommended }\end{array}$ & $\begin{array}{l}\text { It is 'recommended that } \\
\text { obstetrician- } \\
\text { gynaecologists and other } \\
\text { obstetric care providers } \\
\text { screen patients at least } \\
\text { once during the perinatal } \\
\text { period for depression and } \\
\text { anxiety symptoms using } \\
\text { a standardised, validated } \\
\text { tool.' }\end{array}$ & None & $\begin{array}{l}\text { https://insights.ovid. } \\
\text { com/crossref? } \\
\text { an=00006250- } \\
201811000-00042\end{array}$ \\
\hline
\end{tabular}

